\title{
The Heard-Of model: computing in distributed systems with benign faults
}

\author{
Bernadette Charron-Bost · André Schiper
}

Received: 21 July 2006 / Accepted: 22 April 2009 / Published online: 8 July 2009

(C) Springer-Verlag 2009

\begin{abstract}
Problems in fault-tolerant distributed computing have been studied in a variety of models. These models are structured around two central ideas: (1) degree of synchrony and failure model are two independent parameters that determine a particular type of system, (2) the notion of faulty component is helpful and even necessary for the analysis of distributed computations when faults occur. In this work, we question these two basic principles of fault-tolerant distributed computing, and show that it is both possible and worthy to renounce them in the context of benign faults: we present a computational model based only on the notion of transmission faults. In this model, computations evolve in rounds, and messages missed in a round are lost. Only information transmission is represented: for each round $r$ and each process $p$, our model provides the set of processes that $p$ "hears of" at round $r$ (heard-of set), namely the processes from which $p$ receives some message at round $r$. The features of a specific system are thus captured as a whole, just by a predicate over the collection of heard-of sets. We show that our model handles benign failures, be they static or dynamic, permanent or transient, in a unified framework. We demonstrate how this approach leads to shorter and simpler proofs of important results (non-solvability, lower bounds). In particular, we prove that the Consensus problem cannot be generally solved without an implicit and permanent consensus on heard-of
\end{abstract}

A. Schiper's research funded by the Swiss National Science Foundation under grant number 200021-111701 and Hasler Foundation under grant number 2070.

B. Charron-Bost ( $\square)$

École polytechnique, CNRS, 91128 Palaiseau Cédex, France

e-mail: Charron@lix.polytechnique.fr

A. Schiper

EPFL, 1015 Lausanne, Switzerland

e-mail: Andre.Schiper@epfl.ch sets. We also examine Consensus algorithms in our model. In light of this specific agreement problem, we show how our approach allows us to devise new interesting solutions.

Keywords Consensus - Benign fault - Computational model $\cdot$ HO (Heard-Of) model $\cdot$ Transmission fault

\section{Introduction}

Problems in fault-tolerant distributed computing have been studied in a variety of models. Such models are structured around two central ideas:

1. Degree of synchrony and failure model are two independent parameters that determine a particular type of system.

2. The notion of faulty component is helpful and even necessary for the analysis of distributed computations when faults occur.

In this paper we question these two basic principles of faulttolerant distributed computing, and show that it is both possible and worthy to renounce them in the context of benign faults: we present a computational model, suitable for systems with benign failures, which is based only on the notion of transmission fault.

Computations in our model are composed of rounds. In each round, a process sends a message to each process, waits to receive messages from some processes, and then computes a new state. Every message received at some round has been sent at that round. Consequently, any message missed in a round is definitely discarded. Using the terminology of Elrad and Francez [18], a round is a communication-closed-layer. 
Most of the solutions to agreement problems that have been designed for synchronous message-passing systems as well as for partially synchronous or asynchronous ones are structured in rounds (e.g., $[1,5,14,16,17,41])$. However, concerning impossibility results (lower bounds, nonsolvability, ...) in message-passing systems, round-based computational models have been considered almost always for synchronous systems. The reason for that lies in the fact that it is an open question whether round-based models are equivalent to the ones in which late messages are not discarded.

To the best of our knowledge, Dwork et al. [17] were the first to define a round-based model for non synchronous computing. More precisely, they generalized the classical roundbased computational model for synchronous systems to a large class of partially synchronous systems. Then Gafni [22] extended the round-based model to any type of systems. The basic idea in his model is to study how a system evolves round-by-round and to abstract away the implementation of the communication between processes, be it shared-memory or message-passing. The properties of the communication mechanisms and system guarantees are captured as a whole by a single module that is called Round-by-Round Fault Detector (for short RRFD) module. More precisely, at each round $r$ and for each process $p$, the module provides a set of suspected processes from which $p$ will not wait for a message (here, we call messages the pieces of information that are exchanged, whatever the medium of communication is). At this point, only non-transmission of information appears in the model: the reason why a process is suspected is not specified, whether it is due to the fact that the process is late or has crashed. In this way, synchrony degree and failure model are encapsulated in the same abstract entity.

The latter idea seems quite sound since separating synchrony degree and failure model breaks the continuum that naturally exists between them: for example, message asynchrony means that there is no bound on message delays, and message loss corresponds to infinite delays. Moreover, capturing synchrony degree and failures with the same abstraction gives hope for relating different types of systems, in particular synchronous and asynchronous systems.

Unfortunately, this idea is not followed through to the end in [22] since the notion of failure model is underhandedly reintroduced via the one of faulty component. Indeed, the communication medium is implicitly assumed to be reliable (no anomalous delay, no loss) and when process $p$ receives no message from $q$, the latter process is considered to be responsible for the transmission failure ( $q$ is late or has crashed). The so-called RRFD modules only suspect processes, never links. Obviously, this impacts the design and correctness proofs of algorithms: for example, agreement problems are specified in [22] as usual, exempting faulty processes from making a decision.
The RRFD model and its general extension GIRAF ${ }^{1}$ [25] are here influenced by the component failure models proposed in the literature, e.g., $[5,12,19,20,30,36]$. As pointed out in [37], the component failure models "do not fully capture the reality of systems subject to (possibly transient) ubiquitous failures", which are systems in which "faults occur anywhere in the system and, following a failure, normal functioning case resume after finite (although unpredictable) time". Moreover, the principle of a priori blaming some components for transmission failures yields several major problems $[37,38]$. First, it may lead to undesirable conclusions: for example, in the send-omission failure model, the entire system will be considered faulty even if only one message from each process is lost. Hence there is no algorithm in this traditional component failure model that can tolerate such transient failures, how few they may be. Second, it allows faulty processes to have deviant behaviors: in decision problems, it is very common that a faulty process is not obliged to make a decision. Indeed, even in their uniform versions [23] that require coordination among all the processes that decide, including those to which faults are ascribed, decision problems share the same restricted termination clause that exempts faulty processes from making a decision. For example, a process $p$ that is blamed for the non-delivery of a single message - as this transmission fault is rightly or wrongly accounted for an omission from $p$-is allowed to make no decision even if $p$ is blamed for nothing else. Finally, as already observed by Dolev [12], it appears that the real causes of transmission faults, namely sender failure, receiver failure, or link failure, may be actually unknown. Transmission faults are often ascribed to some components in an arbitrary manner that may not correspond to reality.

Moreover, there is no prima facie evidence that the notion of faulty component is really helpful in the analysis of faulttolerant distributed algorithms. We show that our model leads to the development of new conditions guaranteeing the correctness of fault-tolerant algorithms, and to shorter and simpler proofs. This is due to the fact that the notion of faulty component unnecessarily overloads system analysis with non-operational details. In other words, it is sufficient that the model just specifies transmission faults (effects) without accounting for the faulty components (causes).

Santoro and Widmayer $[37,38,40]$ clearly pointed out this issue. They introduced the Transmission Faults model that locates faults without specifying their cause. A transmission fault can be due to a link failure as well as a process failure. Contrary to classical models in which transmission faults involve only messages sent or received by an unknown but static set of processes (the so-called faulty processes), the Transmission Faults model is well-adapted to dynamic

\footnotetext{
1 We would like to thank one of the reviewers for having pointed out the GIRAF model [25].
} 
failures. However, this model is designed only for synchronous systems. Indeed, Santoro and Widmayer showed that dynamic failures in synchronous systems have the same negative effect as asynchronicity. This de facto reintroduces synchrony degree and failure model as two separate parameters of systems. The Transmission Faults model, also known has Dynamic/Mobile Faults model, has also been investigated in $[9,11,21,26,39]$.

\section{Contribution}

Our aim is to develop a computational model for distributed systems that combines the advantages of the RRFD model [22] and the Transmission Faults model [37,38], but avoids their drawbacks. We propose a round-based model, called Heard-Of (HO for short) in which (1) synchrony degree and failure model are encapsulated in the same high-level abstraction, and (2) the notion of faulty component (process or link) has totally disappeared. As a result, the HO model accounts for transmission faults without specifying by whom nor why such faults occur. There is no evidence that this leads to a more powerful model than existing models from a computational viewpoint. However, we prove the semantic effectiveness of the $\mathrm{HO}$ model, and show that it is more elegant and simpler, specially for the design and correctness proofs of fault-tolerant distributed algorithms (see Sect. 5). Indeed, simplicity and conciseness is crucial for model checking [42] and formal verification of algorithms [8].

A computation in the $\mathrm{HO}$ model evolves in rounds. In each round, a process sends a message to all the others, and then waits to receive messages from the other processes. Communication missed in a round is lost. For each round $r$ and each process $p, H O(p, r)$ denotes the set of processes that $p$ has "heard of" at round $r$, namely the processes from which $p$ receives some message at round $r$. A transmission fault from $q$ to $p$ at round $r$ is characterized by the fact that $q$ does not belong to $H O(p, r)$.

Exactly as in the RRFD model [22], the features of a specific system are captured in the $\mathrm{HO}$ model as a whole, just by a predicate over the collection of the $H O(p, r)$ 's, called a communication predicate.

The HO model handles benign failures, be they static or dynamic, permanent or transient, in a unified framework. In particular, the model can naturally represent link failures, contrary to models with failure detectors [5,22]. Indeed, in such models, when the failure detector module indicates to some process $p$ to stop waiting for a message from $q$, this is interpreted as " $q$ is (suspected to be) faulty". Obviously, such an interpretation makes no sense if links may lose messages.

Another feature of the HO model is that contrary to the random model $[1,10]$ or the Failure Detector model, there is no notion of "augmenting" asynchronous systems with external devices (oracles) that processes may query: the communi- cation predicate corresponding to an $\mathrm{HO}$ system is an integral part of the model and should be rather seen as defining the environment [7]. The weaker the predicate of an HO system is, the more freedom the environment allows the system, and the harder it is to solve problems. The $\mathrm{HO}$ abstraction (communication predicates) is supported only by the messages sent in the HO algorithm. In other words, we do not decouple predicates from the underlying algorithms in our approach. This is the reason why we encapsulate algorithm and communication predicate in the same structure that we shall call an $\mathrm{HO}$ machine.

Besides the construction of the HO model, we present various results about the Consensus problem that illustrate its semantic effectiveness. Our first result concerns systems that never partition; it characterizes the minimal communication predicate needed to solve Consensus in such systems. To do so, we first introduce the concept of translation of communication predicates. Informally, a communication predicate $\mathcal{P}$ can be translated into another one $\mathcal{P}^{\prime}$ if there is a distributed algorithm that transforms heard-of sets satisfying $\mathcal{P}$ into new ones satisfying $\mathcal{P}^{\prime}$. Any problem that is solvable under $\mathcal{P}^{\prime}$ is then solvable under $\mathcal{P}$ instead. The so-defined relation is reflexive and transitive, and thus orders communication predicates with respect to their abilities to solve problems. If $\mathcal{P}$ can be translated into $\mathcal{P}^{\prime}$, then we say that $\mathcal{P}$ is at least as strong as $\mathcal{P}^{\prime}$.

Of special interest is the communication predicate $\mathcal{P}_{s p \_u n i f}^{*}$ which guarantees that at each round, all processes hear of the same non-empty subset of processes. Such a permanent operational agreement on heard-of sets clearly suffices to solve Consensus. Conversely and more surprisingly, we show that under the condition that there is no heard-of set partitioning-i.e., at each round, any two processes hear of at least one common process - if Consensus is solvable with the communication predicate $\mathcal{P}$, then $\mathcal{P}$ is at least as strong as $\mathcal{P}_{s p \_u n i f}^{*}$. In other words, Consensus cannot be solved without an implicit permanent agreement on the heard-of sets.

Then we describe four basic translations. Using these translations, we prove several results related to the communication predicate guaranteeing that every round has a non-empty kernel, i.e., at each round there is some process that is heard by all. We show that non-empty kernel rounds can be emulated by majority heard-of sets, and more generally, can be emulated in any system that never partitions. By means of these basic translations, we also give a simple direct proof of the reduction of the worst-case synchronous lower bounds $[15,31]$ to the general FLP asynchronous impossibility result [20] (this reduction has been previously established by Gafni for Atomic-Snapshot asynchronous systems [22]). This exemplifies how, by getting rid of the first principle which artificially separates synchrony degree and failure model, we can describe synchronous and asynchronous systems in a unified framework, and take advantage of this to 
relate impossibility results that are traditionally considered as quite different in essence. ${ }^{2}$

Finally we study how to solve Consensus in systems prone to partitioning. The $\mathrm{HO}$ formalism enables us to describe well-known Consensus algorithms, and also to design new solutions. For each Consensus algorithm, we determine a simple communication predicate which guarantees correctness. Interestingly, all the communication predicates that we display express conditions that have to hold just sporadically, contrary to the perpetual correctness condition " $\Omega$ " in the Failure Detector model $[4,7]$. Hence, the HO model seems to be a natural formalism for expressing fine-grained conditions with respect to time. Moreover, in many real systems, we observe series of "bad" and "good" periods with regard to both synchrony and faults. Since they just require sporadic conditions on heard-of sets, the algorithms that we examine are well-adapted to such systems, and so are quite realistic solutions to the Consensus problem.

This paper is structured as follows. In Sect. 2, we describe our model, and present many traditional systems in the $\mathrm{HO}$ framework. In Sect. 3, we define the notion of translation and propose a characterization of the communication predicates that make Consensus solvable (under certain transmission fault bounds). In Sect. 4, we give four basic translations, and highlight the key role played by the "no partitioning" assumption. In Sect. 5, we describe several Consensus algorithms, and determine $\mathrm{HO}$ conditions for their correctness. Section 6 concludes the paper.

\section{HO model}

As explained in the Introduction, computations in our model are composed of rounds, which are communication-closed layers in the sense that any message sent in a round can be received only at that round. The technical description of computations is similar to the ones in [17,22], and so the model generalizes the classical notion of synchronized rounds developed for synchronous systems [29]. We introduce the notion of kernel of round $r$ that represents what processes share during round $r$ from the operational viewpoint. As we shall show, this notion plays a key role in solving Consensus.

\footnotetext{
2 There is another approach to unify synchronous and asynchronous models, which consists in developing tools for model-independent analysis of decision problems, instead of using translations between system models. More specifically, [24] develops arguments from algebraic topology for synchronous, partially synchronous, and asynchronous systems as well, while [32] introduces the notion of layering as a tool for model-independent analysis of the Consensus problem. Note that the approaches in $[24,32]$ both use the notion of faulty component.
}

\subsection{Heard-of sets and communication predicates}

We suppose that we have a non-empty set $\Pi$ of cardinality $n$, a set of messages $M$, including a null placeholder indicating the empty message. To each $p$ in $\Pi$, we associate a process, which consists of the following components: a set of states denoted by states $_{p}$, a subset init $_{p}$ of initial states, for each positive integer $r$ called round number, a message-sending function $S_{p}^{r}$ mapping state $s_{p} \times \Pi$ to a unique (possibly $n u l l)$ message, and a state-transition function $T_{p}^{r}$ mapping states $_{p}$ and partial vectors (indexed by $\Pi$ ) of elements of $M$ to states $p$. In each round $r$, process $p$ first applies $S_{p}^{r}$ to the current state, emits the "messages" to be sent to each process, and then, for a subset $H O(p, r)$ of $\Pi$ (indicating the processes which $p$ hears of), applies $T_{p}^{r}$ to its current state and the partial vector of incoming messages whose support is $H O(p, r)$. The collection of processes is called an algorithm on $\Pi$.

Computation evolves in an infinite sequence of rounds. For each computation, we determine its heard-of collection which is the collection of subsets of $\Pi$ indexed by $\Pi \times \mathbb{N}^{*}$ :

$(H O(p, r))_{p \in \Pi, r>0}$.

A communication predicate $\mathcal{P}$ is defined to be a predicate over heard-of collections, that is a boolean function over the collections of subsets of $\Pi$ indexed by $\Pi \times \mathbb{N}^{*}$ :

$\mathcal{P}:\left(2^{\Pi}\right)^{\Pi \times \mathbb{N}^{*}} \longrightarrow\{$ false, true $\}$

Since we want to define a non-trivial notion of solvability (see Sect. 2.2), we only consider communication predicates different from the constant predicate "false". Moreover, as we do not want the correctness of algorithms to depend on the time (or round) at which algorithms start to run (see Proposition 1), we restrict ourselves to the class of communication predicates $\mathcal{P}$ whose truth-value is invariant under time translation, i.e., $\mathcal{P}$ has the same truth-value for all the heard-of collections $(H O(p, r+i))_{p \in \Pi, r>0}$, where $i$ is any given integer. Such a communication predicate will be said to be time invariant.

For example, consider some condition $\mathcal{C}$ over the heard-of sets in a given round, that is a predicate over collections of subset of $\Pi$ indexed (only) by $\Pi$ :

$\mathcal{C}:\left(2^{\Pi}\right)^{\Pi} \longrightarrow\{$ false, true $\}$.

Then, the communication predicate

$\mathcal{P}_{(\mathcal{C})}:: \exists r_{0} \geq 0: \mathcal{C}$ holds at $r_{0}$

is not time invariant if $\mathcal{C}$ is not constant, while

$\mathcal{P}_{(\mathcal{C})}{ }^{\infty}:: \forall r>0, \exists r_{0} \geq r: \mathcal{C}$ holds at $r_{0}$,

which expresses that $\mathcal{C}$ holds infinitely often, is a time invariant communication predicate. From now on, the term 
communication predicate will only refer to a non-trivially false and time invariant predicate.

For any round $r$, its kernel is defined as the set of processes

$K(r)=\bigcap_{p \in \Pi} H O(p, r)$.

Intuitively, it consists of the processes which are heard by all in round $r$. More generally, we introduce the kernel $K(\phi)$ of any set $\phi$ of rounds as:

$K(\phi)=\bigcap_{r \in \phi} K(r)$.

When $\phi$ is the set of all the rounds in the computation, this defines the (global) kernel of the computation:

$K=\bigcap_{r>0} K(r)$.

It will be convenient to introduce the cokernel of some round, or more generally of some collection of rounds, as the complement in $\Pi$ of the above defined kernels. Thus, with the same notation as above, we let

$\operatorname{co} K(r)=\Pi \backslash K(r), \operatorname{co} K(\phi)=\Pi \backslash K(\phi), \operatorname{co} K=\Pi \backslash K$.

Round $r$ is said to be uniform when, for any two processes $p, q$ in $\Pi$,

$H O(p, r)=H O(q, r)$.

Round $r$ is said to be a nek (for non-empty kernel) round if

$K(r) \neq \emptyset$,

and it is said to be split when there exist two processes $p, q$ in $\Pi$ such that

$H O(p, r) \cap H O(q, r)=\emptyset$.

Obviously, a nek round is not split, but the converse does not hold. Moreover, a non-trivial uniform round, that is a uniform round with a non-empty common heard-of set, is a nek round.

A nek computation is a computation whose global kernel is non-empty. In such a computation, there is at least one process from which every process hears during the whole computation. A computation is said to be space uniform when each of its rounds is uniform. It is said to be time uniform when the sets $H O(p, r)$ do not vary in time:

$\forall r>0, \forall p \in \Pi: H O(p, r)=H O(p, r+1)$.

Finally, a computation is said to be regular when a process that is not heard by some process at some round is not heard by any process later:

$\forall r>0, \forall p \in \Pi: H O(p, r+1) \subseteq K(r)$.

Note that regularity is a weak form of the combination of space and time uniformity.
Equivalently, we could rather consider talked-to sets, denoted $T T(p, r)$, and defined by

$T T(p, r)=\{q \in \Pi: p \in H O(q, r)\}$,

which are the dual notion of heard-of sets. Contrary to $H O(p, r)$, process $p$ cannot know $T T(p, r)$ at the end of round $r$, and this is the reason why we have preferred to express the communication properties of computations in terms of their heard-of collections instead of their talked-to collections.

\subsection{HO machines}

A Heard-Of machine (or $\mathrm{HO}$ machine for short) for $\Pi$ is a pair $M=(A, \mathcal{P})$ where $A$ is an algorithm on $\Pi$ and $\mathcal{P}$ is a communication predicate. For example, we shall consider the $\mathrm{HO}$ machines with the communication predicate:

$\mathcal{P}_{\text {sp_unif }}:: \forall r>0, \forall p, q \in \Pi^{2}: H O(p, r)=H O(q, r)$,

that is HO machines with space uniform computations, and those with regular computations:

$\mathcal{P}_{\text {reg }}:: \forall r>0, \forall p \in \Pi: H O(p, r+1) \subseteq K(r)$.

We shall also consider the class of HO machines that share the "no split" predicate:

$\mathcal{P}_{\text {nosplit }}:: \forall r>0, \forall p, q \in \Pi^{2}$ :

$$
H O(p, r) \cap H O(q, r) \neq \emptyset,
$$

the subclass of $\mathrm{HO}$ machines with the communication predicate:

$\mathcal{P}_{\text {nekrounds }}:: \forall r>0: K(r) \neq \emptyset$,

and the one with the stronger communication predicate:

$\mathcal{P}_{\text {nek }}:: K \neq \emptyset$.

More generally, we introduce the communication predicate:

$\mathcal{P}_{K}^{f}::|\operatorname{co} K| \leq f$

which is equivalent to

$|K| \geq n-f$.

We shall also consider the weaker predicate:

$\mathcal{P}_{H O}^{f}:: \forall r>0, \forall p \in \Pi:|H O(p, r)| \geq n-f$

and more specifically $\mathcal{P}_{H O}^{m a j}=\mathcal{P}_{H O}^{\left[\frac{n-1}{2}\right]}$ that asserts every heard-of set is a majority set.

A run of $(A, \mathcal{P})$ is totally determined by a set of initial states (one per process) and a heard-of collection that satisfies $\mathcal{P}$. To each run corresponds the collection of the states (one per process and per round) reached by processes during the run; we denote $p$ 's state at the end of round $r$ by $\sigma_{p}^{(r)}$. By extension, the initial state of $p$ is denoted by $\sigma_{p}^{(0)}$. In the 
sequel, given a run of $M$, for any variable $X_{p}$ of process $p$, $X_{p}^{(r)}$ will denote the value of $X_{p}$ after $r$ rounds of this run.

A problem $\Sigma$ for $\Pi$ is a predicate over state collections indexed by $\Pi \times \mathbb{N}^{*}$ :

$\Sigma:\left(s_{p}^{r}\right)_{p \in \Pi, r>0} \longrightarrow \Sigma\left(\left(s_{p}^{r}\right)_{p \in \Pi, r>0}\right) \in\{$ false, true,

where $s_{p}^{r} \in$ states $_{p}$ for every $p \in \Pi$. An HO machine $M=(A, \mathcal{P})$ solves a problem $\Sigma$ if the state collection in each of its runs satisfies $\Sigma$; then we say that problem $\Sigma$ is solvable under $\mathcal{P}$.

Since communication predicates are invariant under time translation, and round numbers are not part of process states, correctness of algorithms does not depend on the time at which algorithms start to run. Formally, for any integer $i$ and any $\mathrm{HO}$ algorithm $A$ with the message-sending and statetransition functions $S_{p}^{r}$ and $T_{p}^{r}$ respectively, let ${ }^{i} A$ denote the algorithm defined by the message-sending functions ${ }^{i} S_{p}^{r}$ and the state-transition functions ${ }^{i} T_{p}^{r}$ such that ${ }^{i} S_{p}^{r}$ and ${ }^{i} T_{p}^{r}$ are trivial for the first $i$ rounds, and for any round $r>i$,

${ }^{i} S_{p}^{r}=S_{p}^{r-i}$ and ${ }^{i} T_{p}^{r}=T_{p}^{r-i}$.

Proposition 1 If the $H O$ machine $M=(A, \mathcal{P})$ solves $\Sigma$, then for any integer $i$, the $\mathrm{HO}$ machine ${ }^{i} M=\left({ }^{i} A, \mathcal{P}\right)$ also solves $\Sigma$.

In this paper, we concentrate on the well-known agreement problem, called Consensus. In this problem, each process $p$ has an initial value $v_{p}$ from a fixed set $V$, and must reach an irrevocable decision on one of the initial values. Thus each value $v$ in $V$ corresponds to an initial state $s_{p}^{v}$ of process $p$, signifying that $p$ 's initial value is $v: \sigma_{p}^{(0)}=s_{p}^{v}$.

Process $p$ has also disjoint sets of decision states $\Sigma_{p}^{v}$, one per value $v$ in $V$, meaning that $p$ has decided $v$. Then the Consensus problem in $V$ is defined as the conjunction of the following requirements:

Irrevocability. Once a process decides a value, it remains decided on that value:

$\forall p \in \Pi, \forall v \in V, \forall r>0$ :

$$
\sigma_{p}^{(r)} \in \Sigma_{p}^{v} \Rightarrow \forall r^{\prime} \geq r: \sigma_{p}^{\left(r^{\prime}\right)} \in \Sigma_{p}^{v}
$$

Agreement. No two processes decide differently: ${ }^{3}$

$$
\begin{aligned}
& \forall p, q \in \Pi, \forall v, w \in V, \forall r, r^{\prime}>0: \\
& \quad \sigma_{p}^{(r)} \in \Sigma_{p}^{v} \wedge \sigma_{q}^{\left(r^{\prime}\right)} \in \Sigma_{q}^{w} \Rightarrow v=w .
\end{aligned}
$$

\footnotetext{
${ }^{3}$ Note that under agreement, irrevocability is equivalent to the following weaker condition:

$\forall p \in \Pi, \forall v \in V, \forall r>0: \sigma_{p}^{(r)} \in \Sigma_{p}^{v} \Rightarrow \forall r^{\prime} \geq r, \exists v^{\prime} \in V: \sigma_{p}^{\left(r^{\prime}\right)} \in \Sigma_{p}^{v^{\prime}}$.
}

This remark will be used in several places in the sequel.
Integrity. Any decision value is the initial value of some process:

$\forall v \in V, \forall p \in \Pi, \forall r>0:$

$$
\sigma_{p}^{(r)} \in \Sigma_{p}^{v} \Rightarrow \exists q \in \Pi: \sigma_{q}^{(0)}=s_{q}^{v} .
$$

Termination. All processes eventually decide:

$\forall p \in \Pi, \exists r_{p}>0, \exists v \in V: \sigma_{p}^{\left(r_{p}\right)} \in \Sigma_{p}^{v}$

Since there is no notion of faulty process in the HO model, a process is never exempted from making a decision. Such a strong liveness requirement may seem unreasonable in two respects. Firstly, it may make Consensus needlessly unsolvable in the sense that the resulting Consensus specification might be unsolvable under some communication predicate $\mathcal{P}$ whereas the classical Consensus problem (with the non-uniform Termination condition) is solvable in the type of systems corresponding to $\mathcal{P}$ (see Table 1 ). The paper shows that this objection does not hold.

Secondly, one may wonder whether an $\mathrm{HO}$ algorithm in which all processes decide can be implemented in systems with processes that are prone to crash failures. The answer is yes. Of course, a process that has crashed takes no steps, and so can make no decision. However, the corresponding $\mathrm{HO}$ process is not heard of any more, and so has no impact on the rest of the computation.

More precisely, let $\mathcal{S}$ be a system with possible process crash failures, and let $\mathcal{P}$ be a communication predicate corresponding to $\mathcal{S}$ (cf. Table 1). Suppose that there is a roundbased computational model for $\mathcal{S}$ with similar notions of algorithm, run of an algorithm, problem, and solvability (e.g., the computational model described in [29] for synchronous systems with crash failures). Let $\Sigma^{\mathcal{S}}$ be a problem in this model, and $\Sigma$ its "entirely uniform" version for the $\mathrm{HO}$ model. Assume that $M=(A, \mathcal{P})$ is an $\mathrm{HO}$ machine solving $\Sigma$. Like any $\mathrm{HO}$ algorithm, $A$ is also an algorithm in the model for $\mathcal{S}$. Since a process in $\mathcal{S}$ that has crashed can take no further step, $\Sigma$ is trivially not solvable in $\mathcal{S}$, and in particular $A$ does not solve $\Sigma$ in $\mathcal{S}$. However, we claim that A solves $\Sigma^{\mathcal{S}}$ :

Proposition 2 If the $H O$ machine $(A, \mathcal{P})$ solves $\Sigma$, then $A$ solves $\Sigma^{\mathcal{S}}$ in $\mathcal{S}$.

We give only the intuitive argument, for lacking of a formal correspondence between $\mathcal{S}$ and $\mathcal{P}$, and between $\Sigma^{\mathcal{S}}$ and $\Sigma$.

Proof (sketched) Let $\rho^{\mathcal{S}}$ be any run of $A$ in $\mathcal{S}$. By mapping any process in $\mathcal{S}$ that crashes at some point $t$ in round $r$ onto an $\mathrm{HO}$ process that is no more heard of after $t$, we easily construct a run $\rho$ of $A$ that extends $\rho^{\mathcal{S}}$, i.e., $\rho^{\mathcal{S}}$ is a subsequence of $\rho$. Since $(A, \mathcal{P})$ solves $\Sigma, \rho$ satisfies $\Sigma$, and so 
the projection $\rho^{\mathcal{S}}$ satisfies $\Sigma^{\mathcal{S}}$, which is some "projection" of $\Sigma$ on the set of correct processes.

In the particular case of Consensus, the above argument shows that there is no problem of transposing an $\mathrm{HO}$ machine solving the entirely uniform Consensus specification in a system $\mathcal{S}$ with possible crash failures: the capability of an $\mathrm{HO}$ process to make a decision (in $\rho$ ) is just not implemented (in $\rho^{\mathcal{S}}$ ) if the corresponding process in $\mathcal{S}$ has crashed.

\subsection{How to guarantee communication predicates}

Obviously, an HO machine is implementable in a system as soon as the corresponding communication predicate can be guaranteed by the system. In Table 1, we go over various classical types of message-passing systems of interest, and we examine the communication predicates that they can guarantee. For each type of system listed in Table 1 except asynchronous systems with initial crash failures, we use several results previously established in $[13,17,22]$. As for asynchronous systems with at most $f$ initial crash failures, they clearly support the communication predicate $\mathcal{P}_{\diamond \text { unif }}^{f}$ defined by:

$$
\begin{aligned}
& \mathcal{P}_{\diamond \text { unif }}^{f}:: \exists r_{0}>0, \exists \Pi_{0} \in 2^{\Pi} \text { s.t. }\left|\Pi_{0}\right| \geq n-f, \\
& \qquad \forall p \in \Pi, \forall r \geq r_{0}: H O(p, r)=\Pi_{0} .
\end{aligned}
$$

Moreover, the positive result by Fischer et al. [20] for initial crash failures shows that in the case of a majority of correct processes $(2 f<n)$, space-time uniformity of the heard-of sets can be achieved from the beginning. ${ }^{4}$ That is, $\mathrm{HO}$ machines with the predicate

$$
\begin{aligned}
\mathcal{P}_{\text {unif }}^{f}:: \exists \Pi_{0} \in 2^{\Pi} \text { s.t. }\left|\Pi_{0}\right| \geq n-f, \\
\forall p \in \Pi, \forall r>0: H O(p, r)=\Pi_{0}
\end{aligned}
$$

can be implemented in any asynchronous system provided a majority of processes is correct.

\section{Communication predicates to solve Consensus}

In this section, we address the fundamental question of determining the computational models in which Consensus is solvable. In terms of the HO model, it consists in identifying the communication predicates for which Consensus is solvable.

We partially answer the question by restricting attention to the class of communication predicates from which nek rounds can be emulated: in this class of $\mathrm{HO}$ models, we prove that permanent space uniformity is a necessary and sufficient condition for solving Consensus. In other words, Consensus

\footnotetext{
$\overline{4}$ The algorithm in [20] ensures agreement on the membership of the initial clique.
}

cannot be solved without an implicit and permanent consensus on heard-of sets.

We start by formalizing what it means for an $\mathrm{HO}$ machine $M=(A, \mathcal{P})$ to emulate a communication predicate $\mathcal{P}^{\prime}$. To do that, we first define the notion of a $k$-round translation from $\mathcal{P}$ to $\mathcal{P}^{\prime}$, and then its generalization to translations that take a non constant number of rounds. Translations of the first type are called uniform translations.

\subsection{Uniform translations}

Let $k$ be any positive integer, and let $A$ be an algorithm that maintains a variable $N e w H_{p}$ at every process $p$, which contains a subset of $\Pi$. We call macro-round $\rho$ the sequence of the $k$ consecutive rounds $k(\rho-1)+1, \ldots, k \rho$. The value of $\mathrm{NewHO}_{p}$ at the end of macro-round $\rho$ is denoted New $\mathrm{HO}_{p}^{(\rho)}$. We say that the HO machine $M=(A, \mathcal{P})$ emulates the communication predicate $\mathcal{P}^{\prime}$ in $k$ rounds if for any run of $M$, the following holds:

E1: If process $q$ belongs to $N e w H O_{p}^{(\rho)}$, then there exist an integer $l$ in $\{1, \ldots, k\}$, a chain of $l+1$ processes $p_{0}, p_{1}, \ldots, p_{l}$ from $p_{0}=q$ to $p_{l}=p$, and a subsequence of $l$ increasing round numbers $r_{1}, \ldots, r_{l}$ in macro-round $\rho$ such that for any index $i, 1 \leq i \leq l$, we have $p_{i-1} \in H O\left(p_{i}, r_{i}\right)$.

E2: The collection $\left(N e w H O_{p}^{(\rho)}\right)_{p \in \Pi, \rho>0}$ satisfies predicate $\mathcal{P}^{\prime}$.

Condition E1 states that if $q$ is in $\mathrm{NewHO}_{p}$ at macro-round $\rho$, then $p$ has actually heard of $q$ during this macro-round through some intermediate processes $p_{1}, \ldots, p_{l-1}$. Hence this condition excludes trivial emulations of $\mathcal{P}^{\prime}$. Condition E2 states that the variables $\mathrm{NewHO}_{p}$ simulate heard-of sets satisfying $\mathcal{P}^{\prime}$. If there exists an algorithm $A$ such that the HO machine $(A, \mathcal{P})$ emulates $\mathcal{P}^{\prime}$ in $k$ rounds, then we write $\mathcal{P} \succeq_{k} \mathcal{P}^{\prime}$, and we say that $A$ is a $k$ round translation of $\mathcal{P}$ into $\mathcal{P}^{\prime}$.

Note that if $\mathcal{P} \Rightarrow \mathcal{P}^{\prime}$, the trivial algorithm in which each process $p$ writes the value of $\mathrm{HO}(p, r)$ into $\mathrm{NewHO}_{p}$ at the end of each round $r$ is a one round translation of $\mathcal{P}$ into $\mathcal{P}^{\prime}$, and so $\mathcal{P} \succeq_{1} \mathcal{P}^{\prime}$.

\subsection{General translations}

Now we generalize the previous definition to translations that take a non-constant number of rounds in time and space. For that, each process $p$ maintains an additional variable MacroRound $p$ initialized to 0. Upon updating $\mathrm{NewHO}_{p}$, process $p$ increments MacroRound $p$ by 1 . When $p$ sends a basic message $m$, it tags $m$ with the current value of MacroRound . Moreover, $p$ ignores any message tagged by 
Table 1 Classical system types and communication predicates

\begin{tabular}{|c|c|}
\hline System type & Communication predicate \\
\hline Synchronous system, reliable links, at most $f$ faulty senders & $|K| \geq n-f$ \\
\hline Synchronous system, at most $f$ omission transmission faults per round [38] & $\forall r>0: \sum_{p \in \Pi}|H O(p, r)| \geq n^{2}-f$ \\
\hline Synchronous system, a block of at most $f$ omission transmission faults [38] & $\forall r>0:|K(r)| \geq n-f$ \\
\hline Synchronous system, reliable links, at most $f$ crash failures & $\begin{array}{l}|K| \geq n-f \\
\wedge \\
\forall p \in \Pi, \forall r>0: H O(p, r+1) \subseteq K(r)\end{array}$ \\
\hline $\begin{array}{l}\text { Synchronous communication, asynchronous processes, reliable links, } \\
\text { atomic send to all, at most } f \text { crash failures [13] }\end{array}$ & $\begin{array}{l}\forall p \in \Pi, \forall r>0:|H O(p, r)| \geq n-f \\
\wedge \\
\forall p, q \in \Pi^{2}, \forall r>0: H O(p, r)=H O(q, r)\end{array}$ \\
\hline $\begin{array}{l}\text { Synchronous communication, asynchronous processes, } \\
\text { reliable links, at most } 1 \text { crash failure [13] }\end{array}$ & $\begin{array}{l}\forall p \in \Pi, \forall r>0: 1 \leq|H O(p, r)| \leq 2 \\
\wedge \\
\forall p, q \in \Pi^{2}, \forall r>0: H O(p, r)=H O(q, r)\end{array}$ \\
\hline Asynchronous system, reliable links, at most $f$ crash failures & $\forall p \in \Pi, \forall r>0:|H O(p, r)| \geq n-f$ \\
\hline Asynchronous system, reliable links, at most $f$ initial crash failures & $\begin{array}{l}\forall p \in \Pi:|H O(p, 1)| \geq n-f \\
\wedge(\forall p \in \Pi, \forall r>0: H O(p, r) \subseteq H O(p, r+1)) \wedge \\
\exists \Pi_{0} \subseteq \Pi, \exists r_{0}>0, \forall p \in \Pi, \forall r>r_{0}: H O(p, r)=\Pi_{0}\end{array}$ \\
\hline Same with $f<n / 2[20]$ & $\begin{array}{l}\exists \Pi_{0} \subseteq \Pi \text { s.t. }\left|\Pi_{0}\right| \geq n-f, \forall p \in \Pi, \forall r>0: \\
H O(p, r)=\Pi_{0}\end{array}$ \\
\hline $\begin{array}{l}\text { Partially synchronous system, eventual reliable links, } \\
\text { at most } f \text { crash failures [17] }\end{array}$ & $\begin{array}{l}\exists \Pi_{0} \subseteq \Pi \text { s.t. }\left|\Pi_{0}\right| \geq n-f, \exists r_{0}>0, \forall p \in \Pi, \forall r>r_{0}: \\
H O(p, r)=\Pi_{0}\end{array}$ \\
\hline
\end{tabular}

an integer different to the current value of MacroRound . Then rephrasing the condition E1 as follows

E1: If process $q$ belongs to $N e w H O_{p}^{(\rho)}$, then there exist a chain of processes $p_{0}, p_{1}, \ldots, p_{l}$ from $p_{0}=q$ to $p_{l}=$ $p$, and a subsequence of $l$ increasing round numbers $r_{1}, \ldots, r_{l}$ such that for any index $i, 1 \leq i \leq l$, we have

$$
\begin{aligned}
& r_{i}<r_{i+1}, \text { MacroRound } p_{i}^{\left(r_{i}\right)}=\rho-1 \text {, and } \\
& p_{i-1} \in H O\left(p_{i}, r_{i}\right) \text {. }
\end{aligned}
$$

yields a general definition of translation.

If there exists an algorithm $A$ such that the $\mathrm{HO}$ machine $(A, \mathcal{P})$ emulates $\mathcal{P}^{\prime}$, we write $\mathcal{P} \succeq \mathcal{P}^{\prime}$, and we say that $A$ translates $\mathcal{P}$ into $\mathcal{P}^{\prime}$. Obviously, the relation $\succeq$ contains all the relations $\succeq_{k}$.

Given an emulation of $\mathcal{P}^{\prime}$ by an $\mathrm{HO}$ machine $(A, \mathcal{P})$, any problem that can be solved with $\mathcal{P}^{\prime}$, can be solved with $\mathcal{P}$ instead. To see this, suppose that the HO machine $\left(B, \mathcal{P}^{\prime}\right)$ solves a problem $\Sigma$. We compose $A$ and $B$ in the following way: each process $p$ executes $B$ with rounds that are "split" by $A$. More precisely, concurrently with $B$, every process $p$ runs $A$, and so (locally) determines what we call " $A$ macro- rounds" and maintains the variable $A . N e w H O_{p}$. The algorithm $B$ at process $p$ is then modified as follows: messages of $A$ during an $A$ macro-round $\rho$ piggyback messages sent by $B$ at round $\rho$, and $p$ computes its new state at the end of macro-round $\rho$ by applying $B$ 's state-transition function at round $\rho$ to (1) its state (with respect to $B$ ) at the beginning of $\rho$ and (2) the partial vector of $B$ 's messages indexed by A.NewHO ${ }_{p}^{(\rho)}$.

Proposition 3 If $\Sigma$ is solvable under $\mathcal{P}^{\prime}$ and $\mathcal{P} \succeq \mathcal{P}^{\prime}$, then $\Sigma$ is solvable under $\mathcal{P}$.

The relation $\succeq$ is clearly transitive; thus it orders communication predicates with respect to their ability to solve problems. If both $\mathcal{P} \succeq \mathcal{P}^{\prime}$ and $\mathcal{P}^{\prime} \succeq \mathcal{P}$ hold, then we say that $\mathcal{P}$ and $\mathcal{P}^{\prime}$ are equivalent, and we denote $\mathcal{P} \simeq \mathcal{P}^{\prime}$.

As we shall see below, an important class of translations are those that preserve kernels. More precisely, we introduce the notion of a kernel preserving translation from $\mathcal{P}$ to $\mathcal{P}^{\prime}$ which is defined as an emulation of $\mathcal{P}^{\prime}$ with an HO machine $M=(A, \mathcal{P})$ such that for any run of $M$, we have:

$\bigcap_{p \in \Pi, r \in \rho_{p}} H O(p, r) \subseteq \bigcap_{p \in \Pi} N e w H O_{p}^{(\rho)}$, 
where $\rho_{p}$ denotes the set of rounds that together form the macro-round $\rho$ on $p$.

\subsection{Consensus and nek rounds}

Let $\mathcal{P}_{s p \_u n i f}^{*}$ denote the communication predicate that guarantees any round to be uniform and non-trivial, that is $\mathcal{P}_{s p \_u n i f}^{*}=\mathcal{P}_{s p \_u n i f} \wedge \mathcal{P}^{*}$ with

$\mathcal{P}^{*}:: \forall r>0, \forall p \in \Pi: H O(p, r) \neq \emptyset$.

Proposition 4 Let $\mathcal{P}$ be a communication predicate such that $\mathcal{P} \succeq \mathcal{P}_{s p \_u n i f}^{*}$. Then for any set of values $V$, there exists an algorithm $A$ such that the $\mathrm{HO}$ machine $(A, \mathcal{P})$ solves Consensus in $V$.

Proof Let $A_{0}$ be an algorithm on $\Pi$ such that $\left(A_{0}, \mathcal{P}\right)$ emulates $\mathcal{P}_{s p \_u n i f}^{*}$, and let us fix an arbitrary order $p_{1}, \ldots, p_{n}$ on $\Pi$. Let $A$ be identical to $A_{0}$, except that

1. at each round, each process sends its knowledge about initial values to all;

2. at the end of the first macro-round, each process decides the initial value of the first process in $\mathrm{New} \mathrm{HO}_{p}$, according to the order $p_{1}, \ldots, p_{n}$.

Note that thanks to E2, every $\mathrm{NewHO}_{p}$ is non-empty at the end of macro-round 1. Moreover, from E1 it follows that the decision rule is well-defined since each process knows the initial values of all the processes in the set $\mathrm{NewHO}_{p}$. Hence the decision rule is well-defined, and termination is satisfied. Integrity is a straightforward consequence of item 2. Agreement follows from E2.

This result can be interestingly compared with the impossibility of Consensus with the communication predicate $\mathcal{P}_{\diamond \text { unif }}^{*}$ (cf. Sect. 2.3). It turns out that eventual space-time uniformity is not sufficient for Consensus, whereas space uniformity alone makes Consensus solvable provided it holds from the beginning.

Conversely, the following proposition shows that in the class of HO machines with nek rounds, space uniformity can be achieved permanently if binary Consensus is solvable. In other words, Consensus is solvable only if there is an implicit permanent agreement on the heard-of sets.

Proposition 5 Let $\mathcal{P}$ be a communication predicate such

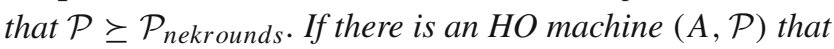
solves binary Consensus, then $\mathcal{P} \succeq \mathcal{P}_{\text {sp_unif }}^{*}$.

Proof Let $B$ be an algorithm that emulates $\mathcal{P}_{\text {nekrounds }}$ from $\mathcal{P}$. From $A$ and $B$, we design an algorithm $C$ and prove that $(C, \mathcal{P})$ emulates $\mathcal{P}_{\text {sp_unif }}^{*}$.

To simulate a macro-round with $C$, every process $p$ first executes one macro-round of $B$ and records the value of
B.NewHO $\mathrm{H}_{p}$ at the end of the macro-round in some variable $\operatorname{Propose}_{p}$. Then it executes $n$ instances of $A$ in parallel, where each solves Consensus (cf. Proposition 1). The initial value of $p$ for the $i$ th instance of $A$ is the truth-value of " $p_{i} \in$ Propose $_{p}$ ". From the decision values, $p$ sets

$$
\begin{aligned}
\text { C.NewHO } & := \\
& \left\{p_{i} \in \Pi:\right. \\
& p \text { decides "true" for the } i \text {-th Consensus }\} .
\end{aligned}
$$

By the agreement condition of Consensus, the emulated macro-round is uniform. Moreover, since $B$ emulates $\mathcal{P}_{\text {nekrounds }}$, there is at least one process $p_{i}$ that belongs to all the $\mathrm{B} . \mathrm{NewHO}$ 's, and so all the initial values for the $i$ th Consensus are equal to "true". By the integrity condition of Consensus, the only possible decision value is "true". In other words, we have $p_{i} \in \bigcap_{p \in \Pi} C$.New HO$O_{p}$. This shows that the emulated uniform macro-round is non-trivial, and so E2 is satisfied.

We now argue E1. Consider a $C$ macro-round (made up of a $B$ macro-round and $n$ executions of $A$ in parallel), and let $p_{i}$ in $C$.New HO $\mathrm{H}_{p}$ at the end of the $C$ macro-round. By the integrity condition of Consensus, there is some processes $x$ such that $p_{i} \in$ Propose $_{x}$. By the Knowledge Transfer theorem [6], for one of them, say $x_{1}$, there is a finite sequence of processes $x_{2}, \ldots, x_{k}=p$ such that during the execution of the $i$ th instance of $A, x_{1}$ sends a message $m_{1}$ to $x_{2}, x_{2}$ sends a message $m_{2}$ to $x_{3}$ after receiving $m_{1}, \ldots, x_{k-1}$ sends a message $m_{k-1}$ to $x_{k}=p$ after receiving $m_{k-2}$. Moreover, since $\mathrm{E} 1$ holds for $B$ macro-rounds, there is a communication path from $p_{i}$ to $x_{1}$ during the first part of the $C$ macro-round. Hence there is a connection from $p_{i}$ to $p$ during the whole $C$ macro-round.

Since binary consensus is trivially reducible to multivalued Consensus, Propositions 4 and 5 provide a characterization of the $\mathrm{HO}$ machines with nek rounds that solve Consensus:

Theorem 1 In the class of communication predicates which are at least as strong as $\mathcal{P}_{\text {nekrounds, }}$, the following assertions are equivalent:

1. For any set $V$, there is an algorithm $A$ such that $(A, \mathcal{P})$ solves Consensus in $V$;

2. $\mathcal{P} \succeq \mathcal{P}_{\text {sp_unif }}^{*}$.

Note that $\mathcal{P}_{s p \_u n i f}^{*}$ is the HO counterpart of one of the system types described in [13], namely the one with asynchronous processes, synchronous communication, reliable links, an atomic send-to-all primitive, and at most $n-1$ crashes. Consequently Theorem 1 shows that, among the system types in [13] for which Consensus is solvable, this special one is the weakest; indeed all of them can emulate nek rounds. 
Fig. 1 Relationships among some basic communication predicates and solvability of Consensus

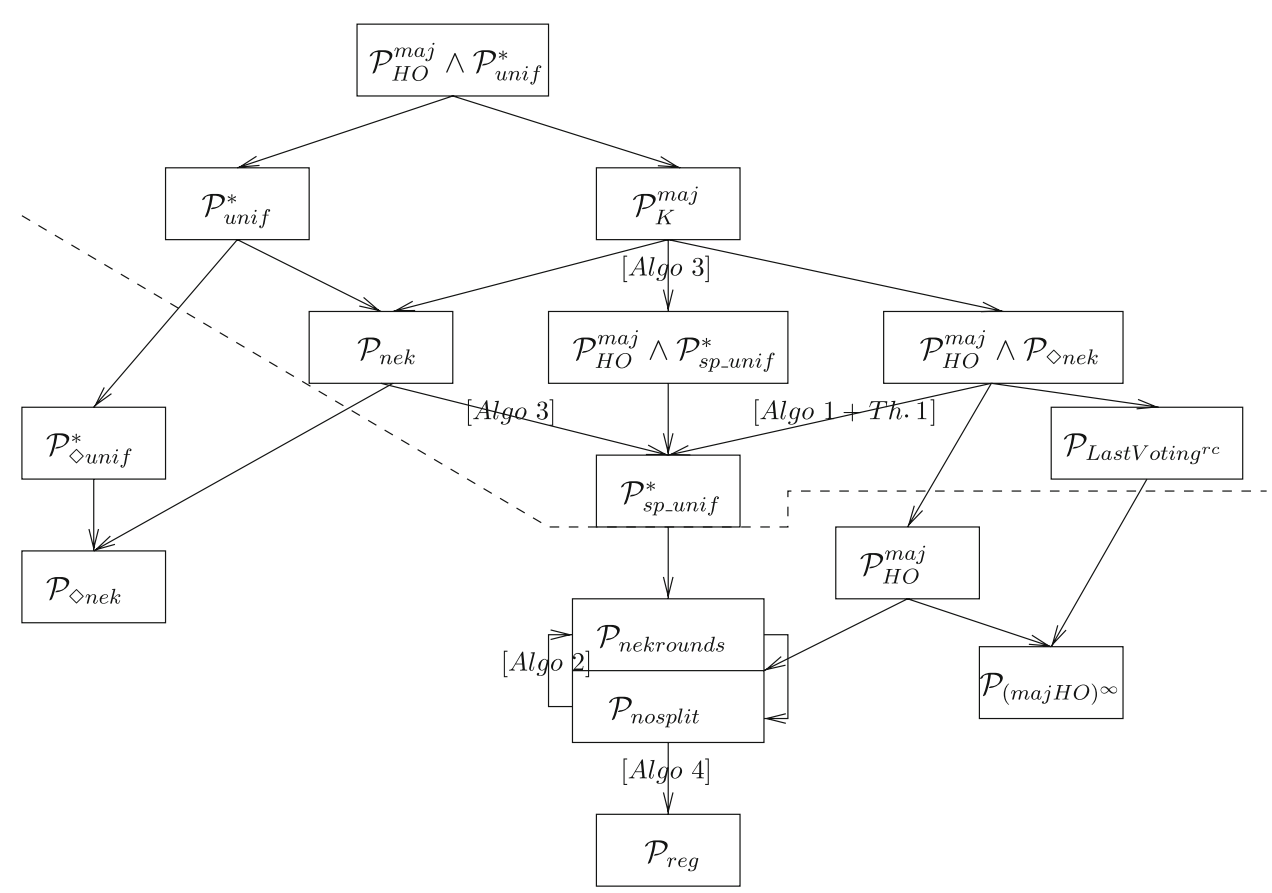

\section{Basic communication predicate translations}

In this section, our aim is to establish some relationships among communication predicates, and to outline a first (partial) map of various classes of these predicates that play a key role for solving Consensus. To do so, we describe several fundamental translations that are all uniform. Such translations simply handle union and intersection of heardof sets. Interestingly, some of them allow us to amplify our characterization of nek round communication predicates that make Consensus solvable. We compare these translations with other ones that have been given in the literature in the context of the classical taxonomy of system types. Our main results are summarized in Fig. 1.

\subsection{A two round translation for increasing kernels}

First we present a two round translation and prove a lower bound on the membership of the (new) kernels of macrorounds. As a result, the translation increases kernels in some significant cases. In particular, it transforms $\mathcal{P}_{H O}^{m a j}$ into $\mathcal{P}_{\text {nekrounds }}$, that is $\mathcal{P}_{H O}^{m a j} \succeq \mathcal{P}_{\text {nekrounds }}$. This translation also provides a direct proof of a very interesting result established by Gafni [22] relating synchronous and asynchronous systems.

The translation computes $N e w H O_{p}^{(\rho)}$ from the collection of heard-of sets in rounds $2 \rho-1$ and $2 \rho$ as follows (see Algorithm 1):

$$
N e w H O_{p}^{(\rho)}:=\bigcup_{q \in H O(p, 2 \rho)} H O(q, 2 \rho-1) .
$$

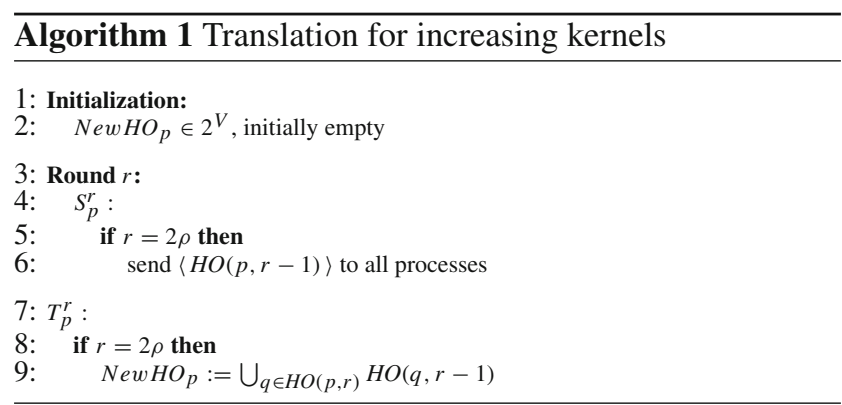

In this way, we emulate a macro-round $\rho$ whose kernel satisfies the following key property:

Proposition 6 If all heard-of sets at rounds $2 \rho-1$ and $2 \rho$ contain at least $n-f_{1}$ and $n-f_{2}$ processes, respectively, then

$\left|\widetilde{K}^{(\rho)}\right| \geq n-f_{1}\left(1+\frac{f_{2}}{n-f_{2}}\right)$,

where $\widetilde{K}^{(\rho)}=\bigcap_{p \in \Pi} N$ New HO$O_{p}^{(\rho)}$.

Proof Consider the directed graph $G_{\rho}$ whose vertices are the processes in $\Pi$, and there is an edge from $p$ to $q$ if and only if $p$ belongs to $H O(q, 2 \rho-1)$. For any vertex $x$ in $G_{\rho}$, let $n b \operatorname{In}(x)$ and $n b O u t(x)$ be the numbers of in-neighbors and out-neighbors of $x$, respectively. The number of edges in $G_{\rho}$ is equal to

$E\left(G_{\rho}\right)=\sum_{x \in \Pi} n b \operatorname{In}(x)=\sum_{y \in \Pi} n b \operatorname{Out}(y)$,

and since $\operatorname{nbIn}(x)=|H O(x, 2 \rho-1)|$, we have

$E\left(G_{\rho}\right) \geq n\left(n-f_{1}\right)$. 
Let us separate the summation $\sum_{y \in \Pi} n b$ Out $(y)$ into those $y$ 's in $\widetilde{K}^{(\rho)}$ and those not in $\widetilde{K}^{(\rho)}$, and let $\widetilde{k}_{\rho}$ denote the cardinality of $\widetilde{K}^{(\rho)}$. Clearly, we have

$$
\sum_{y \in \widetilde{K}^{(\rho)}} n b O u t(y) \leq n \widetilde{k}_{\rho}
$$

For the other term in the sum, we show that for any $y$ that is not in $\widetilde{K}^{(\rho)}$, we have

$n b O u t(y) \leq f_{2}$.

This is true because if $y$ is not in $\widetilde{K}^{(\rho)}$, then there exists some process $p$ such that

$y \notin \bigcup_{q \in H O(p, 2 \rho)} H O(q, 2 \rho-1)$,

that is for any $q$ in $H O(p, 2 \rho), y$ is not in $H O(q, 2 \rho-1)$. In other words, none of the out-neighbors of $y$ in $G_{\rho}$ belongs to $H O(p, 2 \rho)$. Since any heard-of set $H O(p, 2 \rho)$ has at least $n-f_{2}$ elements, $n b O u t(y)$ is at most $f_{2}$. From (1), (2) and (3) it follows that

$n\left(n-f_{1}\right) \leq n \widetilde{k}_{\rho}+\left(n-\widetilde{k}_{\rho}\right) f_{2}$,

and so

$\tilde{k}_{\rho} \geq n-f_{1}\left(1+\frac{f_{2}}{n-f_{2}}\right)$.

With the communication predicate $\mathcal{P}_{H O}^{m a j}=\mathcal{P}_{H O}^{\left[\frac{n-1}{2}\right]}$, Proposition 6 can be specialized as follows:

Corollary 1 There is a two round translation of $\mathcal{P}_{H O}^{m a j}$ into $\mathcal{P}_{\text {nekrounds }}$, and so $\mathcal{P}_{H O}^{m a j} \succeq \mathcal{P}_{\text {nekrounds. }}$.

Proof Take $f_{1}=f_{2}=\left\lceil\frac{n-1}{2}\right\rceil$, which leads to $\widetilde{k}_{\rho} \geq 1$.

Another interesting corollary of Proposition 6 is obtained with $f_{2}=1$ : in this case, Proposition 6 gives

$\widetilde{k}_{\rho} \geq n-f_{1}-\frac{f_{1}}{n-1}$.

Therefore, if $f_{1} \leq n-2$, then we have $\widetilde{k}_{\rho} \geq n-f_{1}$. In particular, in a system with at least 3 processes and heard-of sets of cardinality $n-1\left(f_{1}=f_{2}=1\right)$, we can emulate macro-rounds with kernels of size at least $n-1$, and so the global kernel of $f$ macro-rounds has a membership of over $n-f$ processes. Considering that the communication predicates defined by $\forall r>0, \forall p \in \Pi:|H O(p, r)| \geq n-1$ and $|K| \geq n-f$ are the HO counterparts of asynchronous systems with at most one crash failure and synchronous systems with at most $f$ send omission failures, respectively, we derive the following result relating synchronous and asynchronous systems:
Corollary 2 Asynchronous message-passing systems with at most one crash failure can implement the first $f$ rounds of a synchronous system with at most $f$ send omission failures among $n \geq 3$ processes.

A similar result is shown by Gafni [22] for asynchronous atomic-snapshot shared memory systems with at most one crash failure. Note that the very elegant reduction of the omission failure lower bound to the asynchronous impossibility result [20] that Gafni derives from his result can also be spun off from Corollary 2.

\subsection{Translating no split rounds into nek rounds}

We now show that $\mathcal{P}_{\text {nosplit }}$ and $\mathcal{P}_{\text {nekrounds }}$ are actually equivalent. Clearly, $\mathcal{P}_{\text {nekrounds }}$ implies $\mathcal{P}_{\text {nosplit }}$, and so we have $\mathcal{P}_{\text {nekrounds }} \succeq \mathcal{P}_{\text {nosplit }}$. To prove that $\mathcal{P}_{\text {nosplit }} \succeq \mathcal{P}_{\text {nekrounds }}$, we present a $\lambda(n)$ round translation, where $\lambda(n)$ is the integer satisfying $2^{\lambda(n)-1}<n \leq 2^{\lambda(n)}$, which emulates nek macrorounds from no split rounds. This translation, which appears in Algorithm 2, is an extension from 2 to $\lambda(n)$ of Algorithm 1.

Each macro-round consists of $\lambda(n)$ consecutive rounds. We fix such a macro-round $\rho$, and we denote $r_{1}, \ldots, r_{\lambda(n)}$ the sequence of rounds that form $\rho .{ }^{5}$ Each process $p$ maintains a variable Listen $_{p}$, which is contained in $\Pi$ and is equal to $H O\left(p, r_{1}\right)$ at the end of round $r_{1}$. In the following rounds, $p$ sends the current value of Listen $p$ to all and then computes the new Listen $p$ as the union of the Listen $q$ 's it has just received. That is, at each round $r, r_{2} \leq r \leq r_{\lambda(n)}, p$ sets

$$
\text { Listen }_{p}:=\bigcup_{q \in H O(p, r)} \text { Listen }_{q} .
$$

Theorem 2 Algorithm 2 is a $\lambda(n)$ round translation of $\mathcal{P}_{\text {nosplit into }} \mathcal{P}_{\text {nekrounds, }}$, and so we have $\mathcal{P}_{\text {nosplit }} \simeq$ $\mathcal{P}_{\text {nekrounds. }}$.

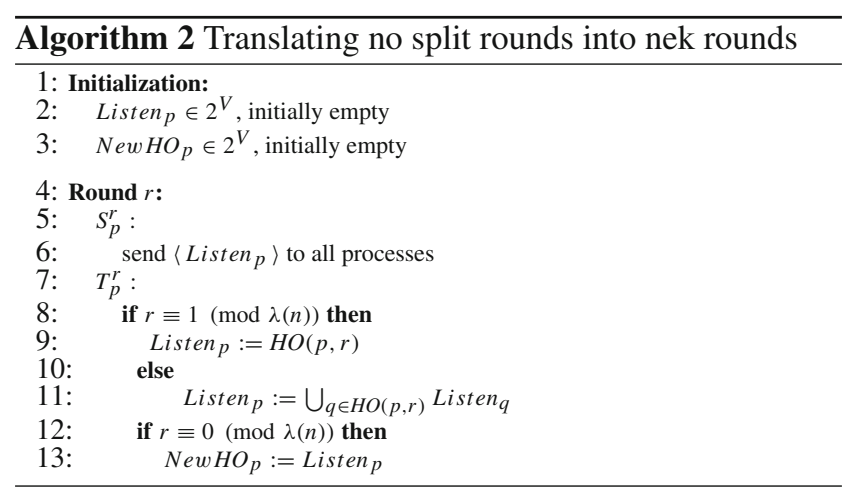

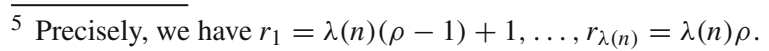


Proof Condition E1 trivially follows from the code of Algorithm 2 (lines 9 and 11). We now prove E2. For that, consider the directed graphs $G_{i}$ induced by the heard-of sets at round $r_{i}$. Let $G_{i}^{*}$ denote the directed graph whose vertices are the processes in $\Pi$, and there is an edge from $p$ to $q$ iff there exists a chain of $i+1$ processes $x_{1}, \ldots, x_{i+1}$ from $x_{1}=p$ to $x_{i+1}=q$ such that

$$
\begin{aligned}
& x_{2} \in H O\left(x_{1}, r_{\lambda(n)}\right), x_{3} \in H O\left(x_{2}, r_{\lambda(n)-1}\right), \ldots, \\
& \text { and } x_{i+1} \in H O\left(x_{i}, r_{\lambda(n)-i+1}\right) .
\end{aligned}
$$

Clearly, $G_{1}^{*}=G_{\lambda(n)}$, and Listen ${ }_{p}$ at the end of round $r_{\lambda(n)}$ is the set of $p$ 's in-neighbours in $G_{\lambda(n)}^{*}$ :

Listen $_{p}^{\left(r_{\lambda(n)}\right)}=\left\{q \in \Pi:(q, p)\right.$ is an edge of $\left.G_{\lambda(n)}^{*}\right\}$.

We now prove the following lemma:

Lemma 1 For any index $i \in\{1, \ldots, \lambda(n)\}$, there is at least one common in-neighbour to any subset of $2^{i}$ processes in the graph $G_{i}^{*}$.

Proof By induction on $i$.

Basis: $i=1$. We have $G_{1}^{*}=G_{\lambda(n)}$, and the lemma coincides with the no split predicate.

Inductive step: Suppose that $i \geq 2$ and the lemma holds in $G_{i-1}^{*}$. Let $\left\{p_{1}, \ldots, p_{2^{i}}\right\}$ be any subset of $2^{i}$ processes. By inductive hypothesis, $p_{1}, \ldots, p_{2^{i-1}}$ have a common inneighbour $x_{1}$ in $G_{i-1}^{*}$, and $p_{2^{i-1}+1}, \ldots, p_{2^{i}}$ have a common in-neighbour $x_{2}$ in $G_{i-1}^{*}$. Since the no split predicate holds at each round, $x_{1}$ and $x_{2}$ have a common in-neighbour in $G_{\lambda(n)-i+1}$, no matter whether $x_{1}=x_{2}$ or not; let $x$ denote this node. By definition of $G_{i}^{*}, x$ is a common in-neighbour to $p_{1}, \ldots, p_{2^{i}}$ in this graph.

$\square_{\text {Lemma } 1}$

Condition E2 directly follows from Lemma 1 as the special case $i=\lambda(n)$ since $n \leq 2^{\lambda(n)}$.

Note that since $\mathcal{P}_{H O}^{m a j}$ implies $\mathcal{P}_{\text {nosplit }}$, Algorithm 2 is a $\lambda(n)$ round translation of $\mathcal{P}_{H O}^{m a j}$ into $\mathcal{P}_{\text {nekrounds }}$. Thus we get another proof of Corollary 1 , but the translation requires $\lambda(n)$ rounds instead of two rounds in Algorithm 1.

Combining Theorems 1 and 2, we get the following corollary:

Corollary 3 Let $\mathcal{P}$ be a communication predicate satisfying $\mathcal{P} \succeq \mathcal{P}_{\text {nosplit. }}$ Then the following assertions are equivalent:

1. There is an algorithm $A$ such that $M=(A, \mathcal{P})$ solves Consensus;

2. $\mathcal{P} \succeq \mathcal{P}_{\text {sp_unif }}^{*}$.

\subsection{A translation achieving space uniformity}

Our third translation achieves space uniformity under the condition of original non-empty global kernels. More precisely, we give a $f+1$ round translation of $\mathcal{P}_{K}^{f}$ into $\mathcal{P}_{s p \_u n i f} \wedge$ $\mathcal{P}_{K}^{f}$.
Processes propagate and collect all the heard-of sets that they have ever seen during $f+1$ consecutive rounds. At the end of the macro-round, $p$ 's new heard-of set is the intersection of the sets of process names that $p$ has just collected at the last round. Formally, each macro-round consists of $f+1$ consecutive rounds. Each process $p$ maintains three variables Listen $_{p}$, Known $_{p}$, and $\mathrm{NewHO}_{p}$, which are all contained in $\Pi$ and are equal to $\Pi,\{p\}$, and $\emptyset$ at the beginning of each macro-round, respectively. At each round, $p$ listens to process $q$ only if it hears of $q$ at all the previous rounds of the macro-round, and so $p$ sets:

Listen $_{p}:=$ Listen $_{p} \cap H O(p, r)$.

Moreover, during the $f$ first rounds of any macro-round, each process $p$ collects the names of all the processes it hears of in its variable $K$ nown $_{p}$; for that, it sends Known no all to processes and then sets:

Known $_{p}:=$ Known $_{p} \cup\left(\bigcup_{q \in \text { Listen }_{p}}\right.$ Known $\left._{q}\right)$.

At the last round of any macro-round, $p$ computes the intersection (instead of the union as in the previous rounds of the macro-round) of the sets Known $q$ it has just collected. The code of the translation is given below (see Algorithm 3).

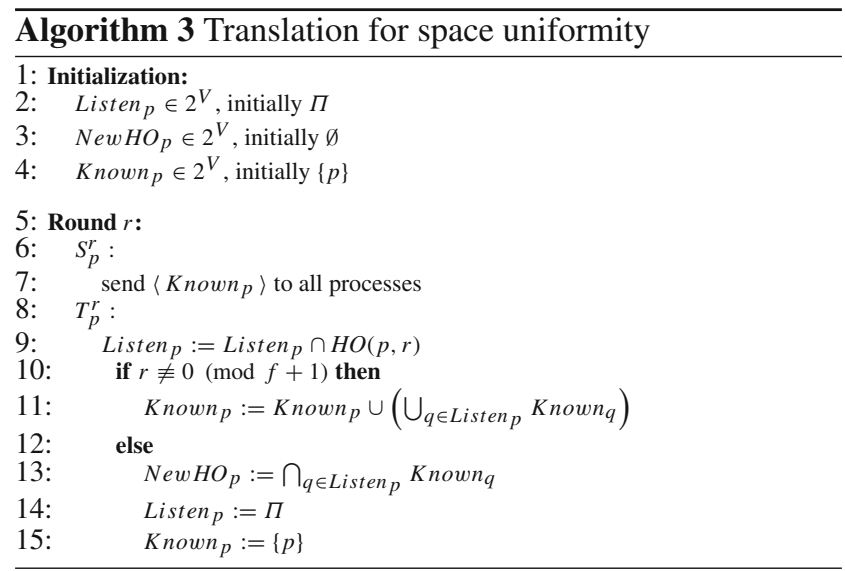

We fix a macro-round $\rho$ and introduce some notation relative to $\rho$. Let $r_{1}, \ldots, r_{f+1}$ denote the sequence of the $f+1$ rounds that form $\rho$. Recall that $K(\rho)$ denotes the kernel of macro-round $\rho$, i.e., $K(\rho)=\bigcap_{r=r_{1}}^{r_{f+1}} K(r)$.

We say that process $p$ knows process $s$ at round $r$ if $s \in$ Known $_{p}^{(r)}$. If $s \in$ Known $_{p}^{(r)} \backslash$ Known $_{p}^{(r-1)}, q \in$ Listen $_{p}^{(r)}$, and $s \in K_{\text {nown }}^{(r-1)}$, then we say that $p$ hears of $s$ from $q$ at round $r$. Finally, process $s$ is said to be good (at macro-round 
$\rho$ ) if $s$ is known by all processes at round $r_{f}$; otherwise $s$ is bad. In other words, the set of good processes is defined by

$$
\text { Good }=\bigcap_{p \in \Pi} \operatorname{Known}_{p}^{\left(r_{f}\right)} .
$$

Thus at line (13), every process computes a local approximation of the set of good processes.

We are going to prove that if $K(\rho)$ contains at least $n-f$ processes, then at the end of any macro-round the New HO's are all identical and contain $K(\rho)$. For that, we start with some preliminary assertions, where as previously, $X_{p}^{(r)}$ denotes the value of $X_{p}$ just at the end of round $r$, except for $X_{p}=$ Listen $_{p}, X_{p}=$ Known $_{p}$, and $r=r_{f+1}$ : in this case, Listen $_{p}^{\left(r_{f+1}\right)}$ and Known ${ }_{p}^{\left(r_{f+1}\right)}$ denote the values of Listen and Known $n_{p}$ in round $r_{f+1}$ just before lines 14 and 15, respectively.

Lemma $2 K(\rho)=\bigcap_{p \in \Pi, r \in\left\{r_{1}, \cdots, r_{f+1}\right\}}$ Listen $_{p}^{(r)}$.

Proof Immediate from the definition of Listen $_{p}^{(r)}$.

Lemma 3 Any process $p$ in $K(\rho)$ is a good process.

Proof Let $p$ be any process in $K(\rho)$. By lines (15) and (11), it follows that all processes know $p$ at the end of round $r_{1}$. Moreover, each Known $_{p}$ variable is non decreasing in each macro-round. This shows that $p$ is a good process.

Lemma 4 If process $p$ hears of some process $s$ at round $r_{k}$, then there exist $k-1$ processes $p_{1}, \ldots, p_{k-1}$, each different from $p$ and $s$, such that $p_{1}$ hears of $s$ from $s$ at round $r_{1}$, $p_{2}$ hears of $s$ from $p_{1}$ at round $r_{2}, \ldots, p_{k-1}$ hears of $s$ from $p_{k-2}$ at round $r_{k-1}$, and $p$ hears of $s$ from $p_{k-1}$ at round $r_{k}$. Moreover, processes $p_{1}, \ldots, p_{k-2}$, and $s$ are all in $\operatorname{coK}(\rho)$.

Proof Since $p$ hears of process $s$ at round $r_{k}$, there exists some process $p_{k-1}$ such that $p_{k-1} \in$ Listen $_{p}^{\left(r_{k}\right)}$ and $s \in$ Known $_{p_{k-1}}^{\left(r_{k-1}\right)}$. Since Listen $_{p}$ is non-increasing, $p_{k-1} \in$ Listen $_{p}^{\left(r_{k-1}\right)}$. This implies that $p_{k-1}$ hears of $s$ at round $r_{k-1}$ since $p$ does not know $s$ at this round. In turn, there exists some process $p_{k-2}$ such that $p_{k-2} \in$ Listen $_{p_{k-1}}^{\left(r_{k-1}\right)}$, and $s \in K_{\text {nown }}^{(k-2)}$. From $s \in K_{p_{k-2}}$ nown $n_{p_{k-2}}^{\left(r_{k-2}\right)}$ and $s \notin$ $\operatorname{Known}_{p}^{\left(r_{k-1}\right)}$, we deduce that $p_{k-2} \notin$ Listen $_{p}^{\left(r_{k-1}\right)}$. By Lemma 2, we have $p_{k-2} \in \operatorname{coK}(\rho)$.

Step by step, we exhibit $k-1$ processes $p_{1}, \ldots, p_{k-1}$ such that for any index $i, 1 \leq i \leq k-1, s \notin$ Known $_{p_{i}}^{\left(r_{i-1}\right)}, s \in$ Known $_{p_{i}}^{\left(r_{i}\right)}$, and $p_{i-1} \in$ Listen $_{p_{i}}^{\left(r_{i}\right)}$.

For any index $i$ such that $2 \leq i \leq k-2$, we have both $s \in \operatorname{Known}_{p_{i-1}}^{\left(r_{i-1}\right)}$ and $s \notin \operatorname{Known}_{p_{i+1}}^{\left(r_{i}\right)}$.
Therefore, $p_{i-1} \notin$ Listen $_{p_{i+1}}^{\left(r_{i}\right)}$. By Lemma 2, we deduce that $p_{i-1}$ belongs to $\operatorname{coK}(\rho)$. Similarly, we have $s \notin \operatorname{Known}_{p_{2}}^{\left(r_{1}\right)}$, and so $s$ belongs to $\operatorname{co} K(\rho)$, too. From $p_{k-2} \in \operatorname{coK}(\rho)$, it follows that all the processes $p_{1}, \ldots, p_{k-2}$, and $s$ are in $\operatorname{coK}(\rho)$.

Lemma 5 If process $p$ knows some bad process $s$ at the end of round $r_{f+1}$, then $p$ has heard of $s$ by the end of the round $r_{f}$, i.e.,

$s \in \operatorname{Known}_{p}^{\left(r_{f+1}\right)} \wedge s \notin \operatorname{Good} \Rightarrow s \in \operatorname{Known}_{p}^{\left(r_{f}\right)}$.

Proof Let $s$ be a bad process; so there exists some process $q$ such that $s \notin$ Known $_{q}^{\left(r_{f}\right)}$. Suppose for contradiction that $p$ hears of $s$ at round $r_{f+1}$. By Lemma 4, there are $f$ processes $p_{1}, \ldots, p_{f}$ each different from both $p$ and $s$ such that $p$ hears of $s$ from $p_{f}$ at round $r_{f+1}$, and processes $p_{1}, \ldots, p_{f-1}$, and $s$ are all in $\operatorname{coK}(\rho)$. Since $s \notin$ Known $_{q}^{\left(r_{f}\right)}$, Listen $_{q}$ contains neither $p$ nor $p_{f}$ at this round. Therefore, $p$ and $p_{f}$ are also in $\operatorname{coK}(\rho)$, which contradicts the fact that $\operatorname{coK}(\rho)$ is of size at most $f$.

Lemma 6 A process is good iff it is known at the end of round $r_{f}$ by some process in the kernel, i.e., $s \in$ Good $\Leftrightarrow$ $\exists p \in K(\rho): s \in$ Known $_{p}^{\left(r_{f}\right)}$.

Proof By definition, a good process is known by all processes at round $r_{f}$.

Conversely, let $s$ be any process known by some process $p$ in $K(\rho)$ at round $r_{f}$. Assume, for the sake of contradiction, that $s$ is bad. Since $p$ is in $K(\rho)$, every process $q$ receives a message from $p$ at round $r_{f+1}$, and so Known $n_{q}$ contains $s$ at the end of round $r_{f+1}$. By Lemma 5 , we deduce that every process already knows $s$ at round $r_{f}$. This contradicts that $s$ is a bad process.

Lemma 7 For any process $p$, at the end of round $r_{f+1}$, New $\mathrm{HO}_{p}$ is composed of all the good processes, i.e., New HO ${ }_{p}^{\left(r_{f+1}\right)}=$ Good.

Proof Obviously, we have Good $\subseteq N e w H O_{p}^{\left(r_{f+1}\right)}$.

Conversely, let $s$ be any process in $N e w \mathrm{HO}_{p}^{\left(r_{f+1}\right)}$; $s$ is known at round $r_{f}$ by all the processes in $\operatorname{Listen}_{p}^{\left(r_{f+1}\right)}$, and in particular by those in $K(\rho)$ by Lemma 2 . From Lemma 6 , it follows that $s$ is a good process since $K(\rho)$ is non-empty.

Lemma 7 says that all the $N e w \mathrm{HO}_{p}$ 's are equal after $f+1$ rounds, and so the collection of the New HO's satisfies $\mathcal{P}_{\text {sp_unif }}$ at the end of each macro-round. Moreover, Lemma 3 implies that the translation preserves kernels, and so emulates $\mathcal{P}_{K}^{f} \wedge \mathcal{P}_{\text {sp_unif }}$. Since E1 is clearly guaranteed, we have proved the following theorem: 
Theorem 3 Algorithm 3 is a $f+1$ translation of $\mathcal{P}_{K}^{f}$ into $\mathcal{P}_{K}^{f} \wedge \mathcal{P}_{\text {sp_unif }}$, and so we have $\mathcal{P}_{K}^{f} \simeq \mathcal{P}_{K}^{f} \wedge \mathcal{P}_{\text {sp_unif }}$.

Combining the latter theorem with Proposition 4, we derive a $f+1$ round algorithm $A$ such that the HO machine $\left(A, \mathcal{P}_{K}^{f}\right)$ solves Consensus. Thus we check that at least for nek machines, the strong termination requirement, namely "every process eventually decides", does not make the Consensus specification harder to solve. Note that the first $f$ rounds of $A$ are identical to those of the FloodSet algorithm [29] which is a well-known Consensus algorithm devised for synchronous systems with at most $f$ crash failures. These two algorithms only differ in round $f+1$ : Algorithm 3 com-

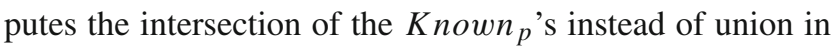
FloodSet. Hence, substituting intersection for union just at the last round is sufficient to guarantee a general agreement among all processes under the only communication predicate $\mathcal{P}_{K}^{f}$ (without regularity).

Interestingly, if we substitute

$\mathrm{NewHO}_{p}:=\mathrm{NewHO}_{p} \cap\left(\cap_{q \in \text { Listen }_{p}}\right.$ Known $\left._{q}\right)$

for

NewHO $\mathrm{N}_{p}:=\bigcap_{q \in \text { Listen }_{p}}$ Known $_{q}$

at line (13) in Algorithm 3, the resulting algorithm translates $\mathcal{P}_{K}^{f}$ into $\mathcal{P}_{K}^{f} \wedge \mathcal{P}_{\text {sp_unif }} \wedge \mathcal{P}_{\text {reg }}$. Considering that the communication predicates $\mathcal{P}_{K}^{f}$ and $\mathcal{P}_{K}^{f} \wedge \mathcal{P}_{\text {reg }}$ are the HO counterparts of synchronous systems with at most $f$ send omission failures and with at most $f$ crash failures respectively, we get an automatic procedure which both guarantees space uniformity and masks send omissions into crash failures.

\subsection{A two round translation for increasing time uniformity}

We now describe a two round translation that increases time uniformity in the sense that it emulates regular runs. This translation can be viewed as a refinement of our first translation (Algorithm 1), with in addition a mechanism for the transition from a macro-round to the next one which guarantees regularity. The basic idea of this mechanism is at each macro-round and for each process to compute an approximation of the kernel of the previous macro-round.

More precisely, each process $p$ maintains a variable Approx $K_{p}$ whose initial value is $\Pi$. At the end of round $2 \rho, p$ sets

$$
\bigcap_{q \in H O(p, 2 \rho)}^{\operatorname{Approx} K_{p}}:=
$$

where Approx $K_{q}^{(\rho-1)}$ denotes the value of Approx $K_{q}$ at the end of the macro-round $\rho-1$. The heard-of set at macro- round $\rho$ for process $p$ is now defined by:

$$
\begin{aligned}
& \operatorname{NewHO}_{p}:= \\
& \bigcup_{q \in H O(p, 2 \rho)}\left(H O(q, 2 \rho-1) \cap \operatorname{Approx} K_{q}^{(\rho-1)}\right) .
\end{aligned}
$$

The resulting algorithm is called Algorithm 4.

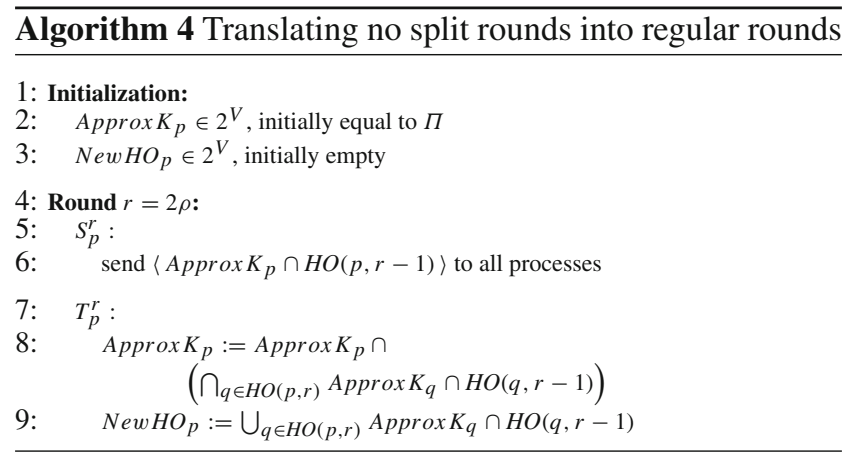

Theorem 4 Algorithm 4 translates $\mathcal{P}_{\text {nosplit }}$ into $\mathcal{P}_{\text {reg }}$, and so we have $\mathcal{P}_{\text {nosplit }} \succeq \mathcal{P}_{\text {reg. }}$. Moreover, it preserves global kernels.

Proof Condition E1 immediately follows from the code of Algorithm 4, line 9. For the same reason, the algorithm preserves kernels. We now prove E2. According to the code of the algorithm, if $x \notin N e w H O_{q}^{(\rho)}$, then for any $s$ in $H O(q, 2 \rho)$, we have $x \notin H O(s, 2 \rho-1) \cap \operatorname{Approx} K_{s}^{(\rho-1)}$.

Because of the no split predicate, for any process $y$, $H O(y, 2 \rho)$ intersects $H O(q, 2 \rho)$, and so there exists $s \in$ $H O(y, 2 \rho)$ such that $x \notin H O(s, 2 \rho-1) \cap A \operatorname{Aprox} K_{s}^{(\rho-1)}$. Hence $x \notin$ Approx $K_{y}^{(\rho)}$. It follows that for any process $p$, $x \notin N e w H_{p}^{(\rho+1)}$. In other words, we have showed that

$\forall p \in \Pi: N e w H O_{p}^{(\rho+1)} \subseteq \bigcap_{q \in \Pi} N e w H O_{q}^{(\rho)}$,

i.e., regularity holds.

Moreover, by definition of Approx $K_{p}$, we easily get

$\forall p \in \Pi: \bigcap_{r=1}^{\rho} K(2 r-1) \subseteq \operatorname{Approx} K_{p}^{(\rho)}$.

It follows that Algorithm 4 preserves global kernels.

From the latter point, we derive the following corollary:

Corollary $4 \forall f \in\{1, \ldots, n-1\}: \mathcal{P}_{K}^{f} \simeq \mathcal{P}_{K}^{f} \wedge \mathcal{P}_{\text {reg }}$.

Since the communication predicates $\mathcal{P}_{K}^{f}$ and $\mathcal{P}_{K}^{f} \wedge \mathcal{P}_{\text {reg }}$ are the $\mathrm{HO}$ counterparts of synchronous systems with at most $f$ send omission failures, and synchronous systems with at most $f$ crash failures, respectively [22], Algorithm 4 provides a general method to convert synchronous algorithms 
tolerant of $f$ crash failures into ones tolerant of $f$ omission senders.

Algorithm 4 is similar to the two round translation given by Neiger and Toueg [33] to mask send omission into crash failures. The only difference between both lies in the processes that are in charge of stopping information transmission: In Neiger and Toueg's algorithm, upon learning it is faulty at some point, process $p$ self censors for the rest of the computation whereas in Algorithm 4, $p$ sends the same messages but the other processes that detect $p$ is faulty do not hear of $p$ anymore.

Algorithms 1 and 4 can be combined into a three round translation that increases both space and time uniformity. In this new translation, each process $p$ inductively computes New $\mathrm{HO}_{p}$ and Approx $K_{p}$ as follows:

$$
\begin{aligned}
& \operatorname{NewHO}(\rho) \\
& \quad \bigcup_{q \in H O(p, 3 \rho)}^{(\rho)}\left(\cup_{s \in H O(q, 3 \rho-1)} H O(s, 3 \rho-2) \cap \operatorname{Approx} K_{s}^{(\rho-1)}\right),
\end{aligned}
$$

and

$$
\begin{aligned}
& \underset{q \in H O(p, 3 \rho)}{\operatorname{Approx} K_{p}}:= \\
& \bigcap_{s \in H O(q, 3 \rho-1)}\left(\cup_{s}(s, 3 \rho-2) \cap \operatorname{Approx} K_{s}^{(\rho-1)}\right) .
\end{aligned}
$$

In this way, the first $f$ rounds of a synchronous system with at most $f$ crash failures can be implemented from an asynchronous message-passing system with at most one crash failure. This three round simulation is identical to the one given by Gafni [22] from asynchronous atomic-snapshot shared memory systems: the last two rounds of the simulation actually correspond to the two round adopt-commit protocol in [22].

\subsection{Comparing communication predicates}

In the previous sections, we saw several interrelationships between some basic communication predicates. These relations and those that are directly derived from the implication relations are illustrated in Fig. 1 as follows: there is a direct edge from $\mathcal{P}$ to $\mathcal{P}^{\prime}$ if $\mathcal{P} \succeq \mathcal{P}^{\prime}$. We adopt the following notation: $\mathcal{P}_{K}^{m a j}=\mathcal{P}_{K}^{\left[\frac{n-1}{2}\right]}$, and

$$
\begin{aligned}
\mathcal{P}_{(\text {maj HO })} & :: \\
& \forall r>0, \exists r_{0} \geq r, \forall p \in \Pi:\left|H O\left(p, r_{0}\right)\right|>n / 2 .
\end{aligned}
$$

The dashed line represents the "Consensus line": a communication predicate $\mathcal{P}$ is above the line iff Consensus is solvable under $\mathcal{P}$. The figure is completed with the predicate $\mathcal{P}_{\text {LastVotingrc }}$ defined in Sect. 5.5.

\section{Consensus and general HO machines}

We now examine general HO machines, some with empty kernel rounds, that solve Consensus. To do so, we first revisit various classical Consensus algorithms devised for asynchronous or partially synchronous systems. For coordinator-based algorithms, we introduce a generalization of $\mathrm{HO}$ machines, the Coordinated $\mathrm{HO}$ machines (or $\mathrm{CHO}$ machines for short).

$\mathrm{HO}$ and $\mathrm{CHO}$ machine formalisms enable us to express well-known Consensus algorithms in a fairly concise and elegant way, and so to extract the algorithmic schemes on which they are based. This not only gives some new insights into these Consensus algorithms, but also allows us to design new ones that are interesting in practice since they are correct under quite realistic conditions. Moreover, it is striking to see how easy it is to determine simple conditions that ensure the correctness of these algorithms from their $\mathrm{HO}$ or $\mathrm{CHO}$ counterparts.

\subsection{A Consensus algorithm à la Ben-Or: the UniformVoting algorithm}

First, we present a Consensus algorithm that to the best of our knowledge, has not yet been described in the literature. It can be viewed as a deterministic version of the Ben-Or algorithm $[1,35]$. We call it the UniformVoting algorithm, see Algorithm 5.

As for all the other algorithms described in Sect. 5, UniformVoting is organized into phases. ${ }^{6}$ A Uniform Voting phase consists of two rounds. Every process $p$ maintains a variable $x_{p}$ containing a value in $V$, initially equal to $p$ 's initial value. Process $p$ broadcasts $x_{p}$ at the first round of each phase, and then adopts the smallest value it has just received. Then, $p$ votes for value $v$ if it has not heard that some process has started the phase with another value; otherwise, $p$ does not cast a vote. At the second round, $p$ sends $v$ or "?" to all, accordingly. In the same message, it sends again the current value of $x_{p}$. If each message that $p$ receives at the second round contains a vote for $v$, then $p$ decides $v$ (this is why we call this algorithm UniformVoting). If $p$ receives some values $v$ different from "?", then it chooses one such value arbitrarily and adopts it for the next phase; otherwise, $p$ adopts the smallest value of the $x_{q}$ 's it has just received.

We now argue that if no round is split, then no process can make a bad decision (agreement). Then we prove that termination is enforced by just one uniform round, which is

\footnotetext{
${ }_{6}$ A phase consists of a fixed number of consecutive rounds. Basically, there is no difference between a phase and a macro-round. We have preferred the term "macro-round" for translations because it seems us more suggestive in this context, but here we use the classical terminology of "phase".
} 


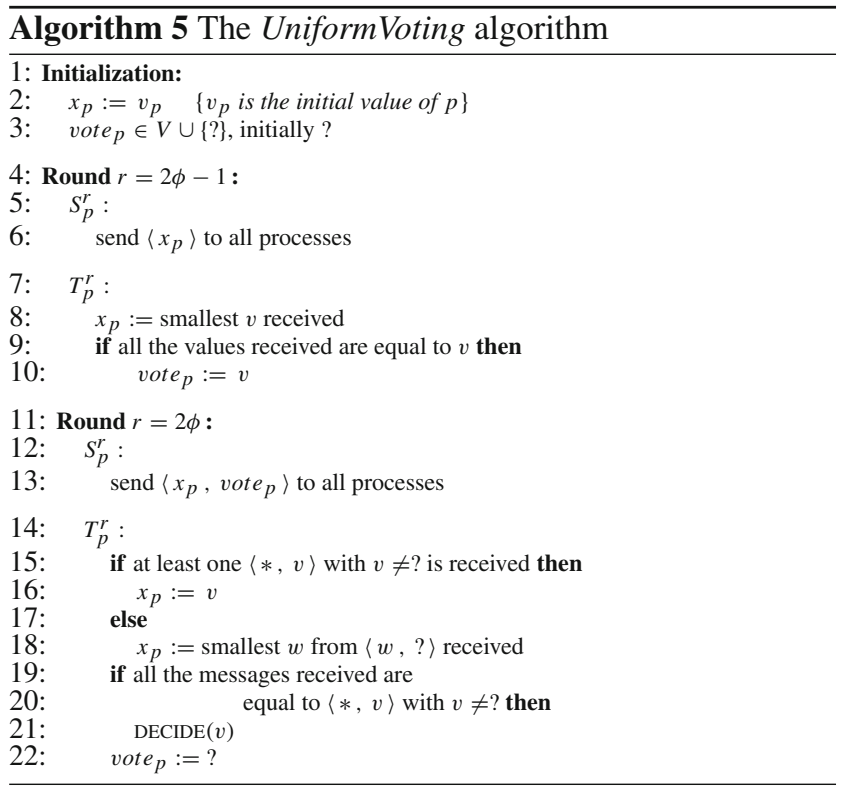

guaranteed by the communication predicate:

$$
\begin{aligned}
\mathcal{P}_{(\text {unif })^{\infty}}:: \forall r>0, \exists r_{0} \geq r, \forall p, q \in \Pi^{2}: \\
\\
H O\left(p, r_{0}\right)=H O\left(q, r_{0}\right) .
\end{aligned}
$$

Theorem 5 The HO machine consisting of the UniformVoting algorithm and the predicate $\mathcal{P}_{\text {nosplit }} \wedge \mathcal{P}_{\text {(unif }^{\infty}}$ solves Consensus.

Proof Integrity is trivially satisfied.

The proof of the agreement condition relies on the fact that if two processes $p$ and $q$ vote for $v$ and $v^{\prime}$ at the same phase, then predicate $\mathcal{P}_{\text {nosplit }}$ ensures that $v=v^{\prime}$. Moreover, predicate $\mathcal{P}_{\text {nosplit }}$ also guarantees that if some process decides $v$ at round $r=2 \phi$, then all the $x_{p}$ 's remain equal to $v$ from round $r$.

For termination, let $r_{0}$ be a uniform round. There are two cases to consider.

1. Round $r_{0}$ is the first round of some phase $\phi_{0}$, i.e., $r_{0}=$ $2 \phi_{0}-1$. Therefore at round $r_{0}$, either all processes vote for the same value $v$ or no process votes.

2. Round $r_{0}$ is the second round of some phase $\phi_{0}$, i.e., $r_{0}=2 \phi_{0}$.

In both cases, all the $x_{p}$ 's are equal at the end of round $2 \phi_{0}$, and every process has decided at the end of round $2 \phi_{0}+2$. It follows that $\mathcal{P}_{\text {(unif }^{\infty}}{ }^{\infty}$, which is invariant by time translation (cf. Sect. 2.1) and guarantees one uniform round, enforces termination of the UniformVoting algorithm.

\subsection{Coordinated HO machines}

Numerous algorithms for Consensus are coordinator-based (e.g., the Consensus algorithms proposed by Dwork et al. [17], Chandra and Toueg's algorithm [5], Paxos [27]). The correctness of these algorithms is guaranteed by some properties on coordinators: for example, termination in $\mathrm{Pa}$ xos requires that during some phase, all processes hear of the coordinator of the phase. For such algorithms, we introduce the Coordinated $\mathrm{HO}$ machine (or $\mathrm{CHO}$ machine for short) for which algorithms refer to the notion of coordinators, and predicates deal not only with heard-of sets, but also with coordinators.

A CHO machine is a pair $M^{c}=(A, P)$ much like the ordinary HO machine. Reflecting the fact that the messages sent by a process $p$ in a round of a CHO machine do not uniquely depend on the current state, but also on the identity of a coordinator, the message-sending function $S_{p}^{r}$ is no longer a function from states $p \times \Pi$ to $M$ but instead a function

$S_{p}^{r}: \Pi \times$ states $_{p} \times \Pi \longrightarrow M$.

Similarly, the state of process $p$ at the end of a round does not only depend on its current state and the collection of the messages it has just received, but also on the identity of its coordinator. So, the transition function $T_{p}^{r}$ is now a function

$T_{p}^{r}:$ states $_{p} \times\left(M^{\Pi}\right)^{*} \times \Pi \longrightarrow$ states $_{p}$

where $\left(M^{\Pi}\right)^{*}$ denotes the set of partial vectors of elements of $M$, indexed by $\Pi$. The functions $\left(S_{p}^{r}\right)_{r>0}$ and $\left(T_{p}^{r}\right)_{r>0}$ define the coordinated process $p$, and the collection of coordinated processes is called a coordinated algorithm.

As for HO machines, at every round $r$, each process $p$ (1) applies the message-sending function $S_{p}^{r}$ to the current coordinator and the current state to generate the messages to be sent, and (2) applies the state-transition $T_{p}^{r}$ to the current state and the incoming messages. The combination of the two steps is called a coordinated round, and $p$ 's coordinator at $r$ is denoted Coord $(p, r)$. Process $p$ sets out to be its coordinator if $p=\operatorname{Coord}(p, r)$.

We say that $r$ is a uniformly coordinated round if

$\forall p, q \in \Pi: \operatorname{Coord}(p, r)=\operatorname{Coord}(q, r)$

and $r$ is well coordinated if

$\forall p \in \Pi: \operatorname{Coord}(p, r) \in H O(p, r)$.

As we shall see in the next sections, uniformly and well coordinated rounds play a key role for guaranteeing correctness of coordinated Consensus algorithms.

A computation of a $\mathrm{CHO}$ machine is uniformly coordinated from round $r_{0}$ if any round $r, r \geq r_{0}$, is uniformly coordinated; a computation is uniformly coordinated if it is uniformly coordinated from the first round.

Usually, when algorithms are decomposed into phases, every process keeps the same coordinator during each whole 
phase. The coordinator of process $p$ during phase $\phi$ can thus be denoted by $\operatorname{Coord}(p, \phi)$.

Given a set of initial states, one per process, each run of a CHO machine is not uniquely determined by the heard-of set collection, but also by the coordinator collection according to space and time, namely $(\operatorname{Coord}(p, r))_{p \in \Pi, r>0}$. A CHO machine for $\Pi$ consists of a coordinated algorithm $A$ and a predicate over both heard-of sets and coordinator collections, called a communication-coordinator predicate, which is invariant by time translation. For example, we shall consider $\mathrm{CHO}$ machines with the predicate:

$$
\begin{aligned}
& \forall r>0, \exists r_{0} \geq r, \forall p, q \in \Pi^{2}: \\
& \quad \operatorname{Coord}\left(p, r_{0}\right)=\operatorname{Coord}\left(q, r_{0}\right) \wedge \operatorname{Coord}\left(p, r_{0}\right) \in H O\left(p, r_{0}\right)
\end{aligned}
$$

or equivalently,

$$
\begin{aligned}
& \forall r>0, \exists r_{0} \geq r, \forall p, q \in \Pi^{2}: \\
& \quad \operatorname{Coord}\left(p, r_{0}\right)=\operatorname{Coord}\left(q, r_{0}\right) \wedge \operatorname{Coord}\left(p, r_{0}\right) \in K\left(r_{0}\right) .
\end{aligned}
$$

Finally, the notion of what it means for a $\mathrm{CHO}$ machine to solve a problem is similar to the one for an $\mathrm{HO}$ machine.

Similarly to the heard-of sets, the way processes determine the name of their coordinators is not specified in the HO formalism: it may be the result of some computation (in other words, the $\mathrm{CHO}$ machine is emulated by an ordinary HO machine), or processes may use some external devices (physical devices or oracles) that are capable of reporting the name of a coordinator to each process. Most of the $\mathrm{CHO}$ machines that we shall consider can be simulated by ordinary $\mathrm{HO}$ machines, and so this generalization does not seem to lead to a more powerful computational model (as explained above, the basic motivation for introducing $\mathrm{CHO}$ machines is to devise Consensus algorithms and to state conditions for their correctness in a simpler way). In particular, we can adopt an "off-line" strategy, usually called the rotating coordinator strategy, which consists in selecting $\operatorname{Coord}(p, r)=$ $p_{1+r} \bmod n$ for every process $p$ in $\Pi$.

When $\Pi=\left\{p_{1}, \ldots, p_{n}\right\}$. Note that fixing the rotating coordinator strategy, any $\mathrm{CHO}$ machine reduces to an $\mathrm{HO}$ machine.

With the rotating coordinator strategy, agreement on the name of a coordinator is for free, that is every round is uniformly coordinated. On the other hand, the on-line strategy that consists in selecting $p$ 's coordinator in its heard-of set provides well coordinated rounds for free (in the case heardof sets do not vary "too much" in time). A critical point is to achieve rounds which are both uniformly and well coordinated.

\subsection{The CoordUniformVoting machine for Consensus}

When looking closer at the UniformVoting machine, we may think to ensure uniformity of one round, and so termination, by the help of coordinators: at the beginning of each phase, coordinators are in charge to make the $x_{p}$ 's values uniform. More precisely, to each phase $\phi$, we add a preliminary round in which every process $p$ that sets out to be coordinator of phase $\phi$ (i.e., $p=\operatorname{Coor} d(p, \phi))$ broadcasts the value of its variable $x_{p}$. Upon receiving a message with value $v$ from $\operatorname{Coord}(q, \phi)$, process $q$ adopts this value for $x_{q}$. Actually, the additional round allows us to simplify the two rounds of UniformVoting: each process $p$ just sends its vote instead of sending both its vote and the value of $x_{p}$. This yields an algorithm that we call CoordUniformVoting (see Algorithm 6).

Since the decision of one process at some round $2 \phi$ of UniformVoting entails that all the $x_{p}$ 's are equal, CoordUniformVoting still satisfies integrity and agreement. If at some phase $\phi_{0}$, all processes agree on some coordinator's name $c$, and this coordinator is in the kernel of $\phi_{0}$, then every process hears of and adopts $x_{c}$ 's value. In that case, all processes decide on this value at the end of phase $\phi_{0}$. This proves the following theorem:

Theorem 6 The CHO machine consisting of the CoordUniformVoting algorithm and the predicate that guarantees no split round and uniformly and well coordinated phases infinitely often solves Consensus.

This exemplifies how the use of coordinators transforms the requirement of a non-trivial uniform round into the one of agreeing on the name of some process in the kernel. Note that agreement on the coordinator of a phase $\phi$ may be achieved by a leader election algorithm. At that point, the question is whether the elected process is actually in the kernel of round $3 \phi-2$.

Instead of using a leader election algorithm, we can adopt the rotating coordinator strategy. We denote by CoordUniformVoting ${ }^{r c}$ the resulting algorithm. With such a coordinator strategy, having the leader in the kernel at some point in computation is ensured by

$\exists \phi_{0}>0: \bigcap_{i=1}^{n} K\left(\phi_{0}+i\right) \neq \emptyset$.

Thus using the rotating coordinator strategy, we substitute uniformity of one round in the UniformVoting machine for some "temporal stability" guaranteeing a sufficiently long period with a non-empty kernel.

Algorithm 6 can be simplified by observing that lines 9 to 16 can be removed, if at line 17 the value received is assigned to vote $_{p}$ (see Algorithm 7).

\subsection{The $D L S$ algorithm}

The algorithms described up to now work correctly only if some invariant properties for the $\mathrm{HO}$ 's are satisfied (e.g., $\mathcal{P}_{\text {nekrounds }}$ or $\mathcal{P}_{\text {nosplit }}$ ). Taking a closer look at these algorithms, it turns out that the safety conditions of Consensus 

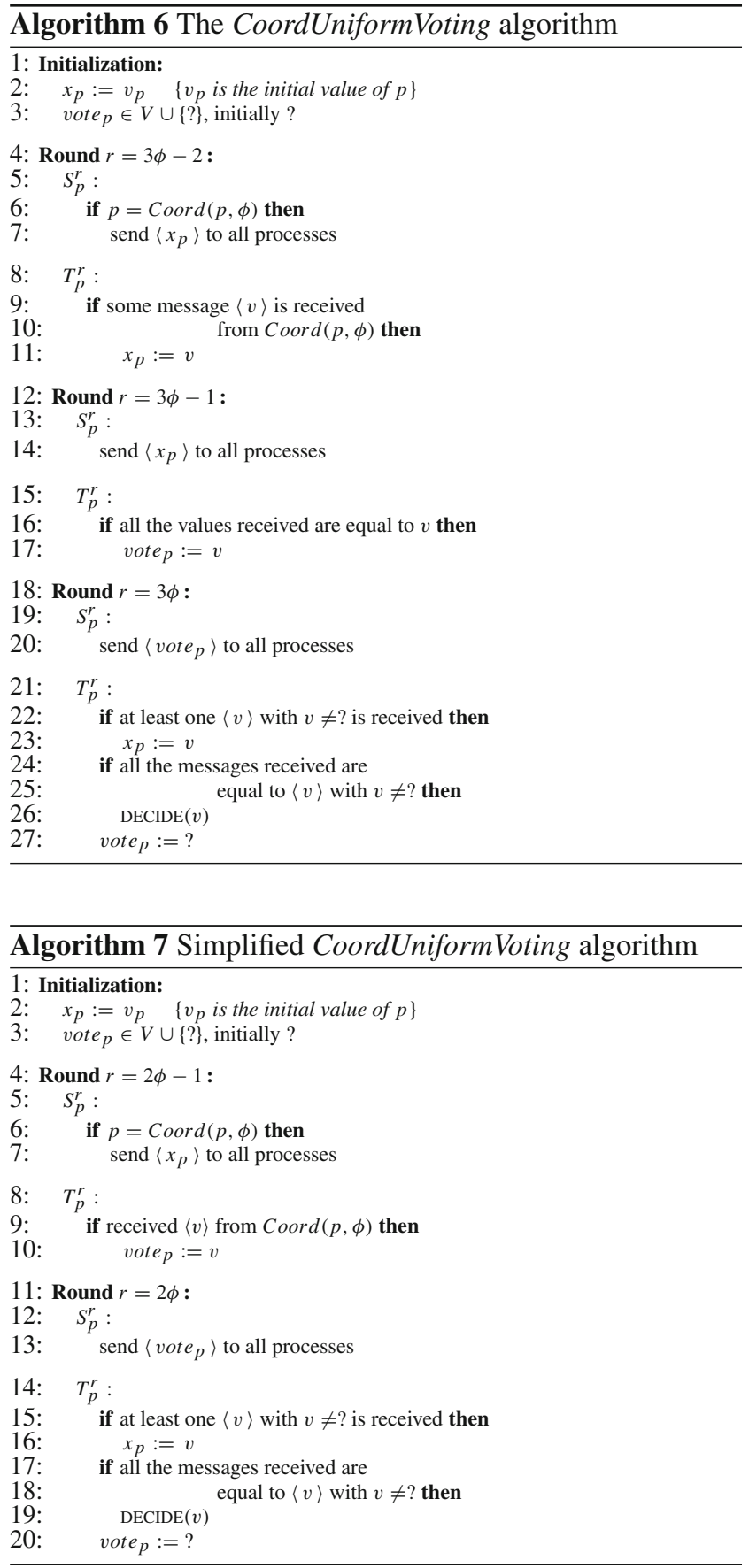

may be violated if there are some "bad" periods during which these predicates do not hold. Thereby, such algorithms cannot be used in systems with message losses (even very rare), which considerably limits the scope of these Consensus algorithms.

In a seminal paper [17], Dwork, Lynch, and Stockmeyer showed how to cope with such bad periods, and designed an algorithm, that we call $D L S$, which solves Consensus if a "sufficiently long" good period occurs. The basic idea of this algorithm is to satisfy safety conditions no matter how badly processes communicate, that is even if many failures occur in the system.

The $D L S$ algorithm was originally described in an HO-like style [17]. Rounds are grouped into phases, where each phase $\phi$ consists of four rounds. The algorithm includes the rotating coordinator strategy, and so each phase $\phi$ is led by a unique coordinator. We refer the reader to [17] for the complete description of DLS.

Dwork et al. [17] proved that their algorithm never violates integrity and agreement. Moreover, they showed that it terminates if "there is a majority of correct processes, and there exists some round GST, such that all messages sent from correct processors at round GST or afterwards are delivered during the round at which they were sent." When shifting GST after the last time a process crashes, the above condition actually corresponds to eventual space-time uniformity:

$$
\begin{aligned}
& \exists \mathrm{GST}>0, \exists \Pi_{0} \text { s.t. }\left|\Pi_{0}\right|>n / 2: \\
& \quad \forall p \in \Pi, \forall r \geq \mathrm{GST}: H O(p, r)=\Pi_{0}
\end{aligned}
$$

which in terms of failure detectors coincides with $\diamond \mathcal{P}$ [5].

As already mentioned in [17], this termination requirement can be drastically weakened: a single well coordinated phase without "too many" transmission failures entails termination. Formally, the DLS algorithm is still correct when replacing eventual space-time uniformity by the following communication predicate:

$$
\begin{aligned}
& \forall \phi>0, \exists \phi_{0} \geq \phi, \exists \Pi_{0} \text { s.t. }\left|\Pi_{0}\right|>n / 2: \\
& \quad\left(\forall p \in \Pi, \forall r \in \phi_{0}: H O(p, r)=\Pi_{0}\right) \wedge\left(p_{1+\phi_{0} \bmod n} \in \Pi_{0}\right) .
\end{aligned}
$$

Variant of DLS: Interestingly, the safety conditions of Consensus, namely integrity and agreement, still hold for any coordinator strategy, even when several processes lead the same phase. In other words, the $\mathrm{CHO}$ extension of $D L S$, that we denote $\operatorname{CoordDLS}$, also satisfies integrity and agreement whatever the communication-coordinator predicate we consider. For termination, we just have to substitute the condition

$$
\begin{aligned}
\left(\forall p, q \in \Pi^{2}: \operatorname{Coord}\left(p, \phi_{0}\right)\right. & \left.=\operatorname{Coord}\left(q, \phi_{0}\right)\right) \wedge \\
(\forall p & \left.\in \Pi: \operatorname{Coord}\left(p, \phi_{0}\right) \in \Pi_{0}\right) .
\end{aligned}
$$

for the condition $p_{1+\phi_{0} \bmod n} \in \Pi_{0}$ in the above communication predicate. Thus this variant of $D L S$ solves Consensus under the condition that there exists some uniform phase ${ }^{7} \phi_{0}$ whose kernel $K\left(\phi_{0}\right)$ is a majority set, and which is led by a single process (coordinator) in $K\left(\phi_{0}\right)$.

\footnotetext{
${ }^{7}$ By uniform phase, we mean that each round of this phase is uniform.
} 


\subsection{A CHO algorithm "à la Paxos"}

The $D L S$ algorithm is based on the rotating coordinator paradigm, which ensures permanent agreement on the coordinator, but as mentioned above, it supports a more flexible coordinator strategy. The idea of using various policies for determining coordinators has been introduced by Lamport in the Paxos algorithm [27]. However, the idea is not followed through to the end in the latter algorithm: the first round of Paxos enforces the choice of a unique coordinator for the remaining rounds of the phase, and so the "Consensus core" in Paxos actually manages a single coordinator per phase.

We have observed here that Paxos is still safe even in the presence of multiple coordinators in the same phase. Indeed, multiple coordinators in the same phase can only prevent casting a vote, since this requires a coordinator to get a majority of (non-null) messages. By the majority condition, at most one coordinator per phase is able to cast a vote. Thus we design a new $\mathrm{CHO}$ algorithm, called LastVoting (Algorithm 8), which follows the basic line of Paxos, but manages possible multiple coordinators per phase. LastVoting is structured as Paxos, except the first round that is removed. This is because LastVoting proceeds in numerically consecutive phases, and so a phase automatically gets a chance to complete. Note that the first two rounds of every phase in Paxos are needed only when the coordinator changes. We always remove the first of these two rounds. The second of these two rounds is needed in LastVoting only when the coordinator changes. Agreement on a single coordinator (also called leader) is not achieved by the algorithm anymore, but is part of the conditions that guarantee termination.

The Consensus core in Paxos shares many common features with $D L S$ : as for $D L S$, the coordinator of a Paxos phase does not cast a vote and misses its turn if it does not receive conclusive information from enough (namely a majority of the) processes. This is the basic reason why $D L S$ and $P a$ xos both tolerate link failures. On the other hand, the two algorithms differ in the values of the coordinators' votes. In $D L S$, the coordinator of a phase votes for some value $v$ if $v$ is a majority value (i.e., it has heard that at least $n / 2$ processes find $v$ acceptable) whereas the coordinator of a Paxos phase votes for the most recent value it has heard of. This is why the coordinator of a Paxos phase can cast a vote (and so can make a decision) even if the preceding round is not uniform. In that respect, our LastVoting algorithm is similar to Paxos.

In the following theorem, we show that LastVoting is always safe (even in the presence of multiple coordinators at some phases), and we exhibit a very simple condition that enforces termination. Interestingly, the latter condition only involves one phase, and the corresponding communicationcoordinator predicate is non-stable, contrary to the " $\Omega$ condition"-classically supposed for Paxos termination-that

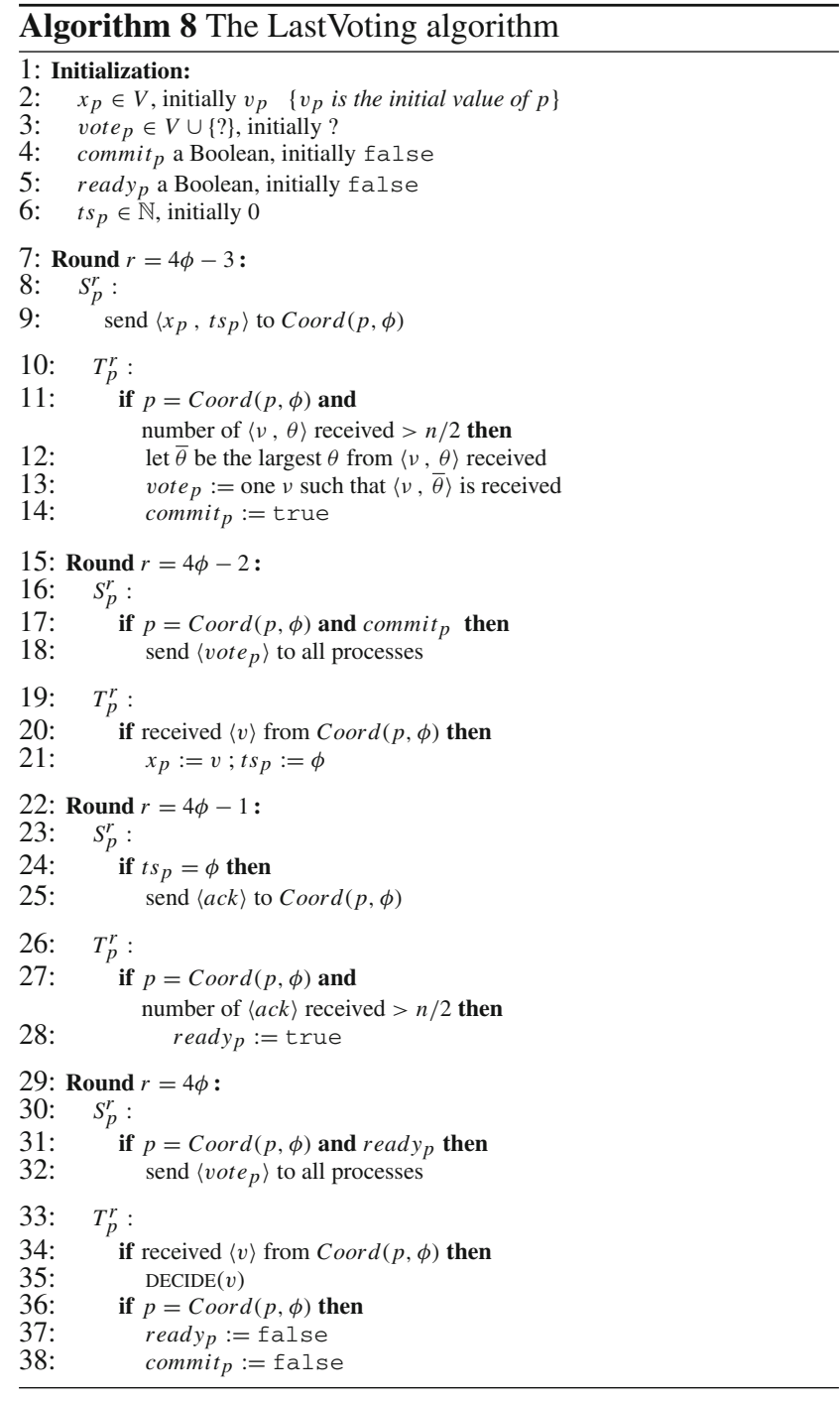

requires uniformly and well coordinated phases permanently from some point in the computation [7].

Theorem 7 The HO machine that consists of the LastVoting algorithm and the communication-coordinator predicate:

$\forall \phi>0, \exists \phi_{0} \geq \phi, \exists c_{0} \in \Pi, \forall p \in \Pi:$
$\left\{\begin{array}{l}\left|H O\left(c_{0}, 4 \phi_{0}-3\right)\right|>n / 2 \wedge\left|H O\left(c_{0}, 4 \phi_{0}-1\right)\right|>n / 2 \\ \wedge \\ \left(\operatorname{Coord}\left(p, \phi_{0}\right)=c_{0}\right) \wedge\left(\operatorname{Coord}\left(p, \phi_{0}\right) \in K\left(\phi_{0}\right)\right)\end{array}\right.$

solves Consensus.

Proof The above communication-coordinator predicate enforces termination of the LastVoting algorithm. Indeed, if the predicate holds, the conditions of lines 17, 27, and 34 are all true at phase $\phi_{0}$.

Integrity follows from the fact that the decision is on vote $_{p}$, that vote $e_{p}$ is always set to one of the $x_{p}$ values received (line 13), and that $x_{p}$ is only updated with vote $p$ received (line 21). 
For agreement, let $\phi_{1}$ be the first phase at which some process makes a decision. Let $p$ be such a process and let $v$ be its decision value. Lines 27 and 31 imply that $p$ 's coordinator at phase $\phi_{1}$, denoted $c$, has received more than $n / 2$ acknowledgements at round $4 \phi_{1}-1$ and vot $e_{c}^{\left(4 \phi_{1}-1\right)}=v$. Moreover, $c$ has received more than $n / 2$ non-null messages at round $4 \phi_{1}-3$.

For any phase $\phi \geq \phi_{1}$, let $\Pi_{\phi}$ denote the set of processes that have updated their timestamp variables at least once since phase $\phi_{1}$ :

$\Pi_{\phi}=\left\{q \in \Pi: t s_{q}^{(\phi)} \geq \phi_{1}\right\}$.

The heart of the proof is the following lemma, which says that from phase $\phi_{1}$, each process $q$ may barter the value of $x_{q}$ only for $v$.

Lemma 8 At any phase $\phi \geq \phi_{1}$, the following holds:

1. $\Pi_{\phi}$ is a majority set, i.e., $\left|\Pi_{\phi}\right|>n / 2$.

2. For any process $q$ in $\Pi_{\phi}$, we have $x_{q}^{(4 \phi-2)}=v$.

Proof By induction on $\phi-\phi_{1}$.

Basis: $\phi=\phi_{1}$. Any process $q$ in $\Pi_{\phi_{1}}$ executes line (21), and so has received a vote from its coordinator $c^{\prime}=\operatorname{Coor} d\left(q, \phi_{1}\right)$. Hence $c^{\prime}$ casts a vote at round $4 \phi_{1}-3$, and so $c^{\prime}$ receives more than $n / 2$ non-null messages at this round. Since each process sends at most one non-null message at round $4 \phi_{1}-3$, we have $c^{\prime}=c$. It follows that

$x_{q}^{\left(4 \phi_{1}-2\right)}=$ vote $_{c}^{\left(4 \phi_{1}-3\right)}=v$.

Moreover, $c$ receives more than $n / 2$ acknowledgements, and so more than $n / 2$ processes execute line 21 at phase $\phi_{1}$. This shows that $\Pi_{\phi_{1}}$ is a majority set.

Inductive step: Suppose that $\phi>\phi_{1},\left|\Pi_{\phi-1}\right|>n / 2$, and for any $q \in \Pi_{\phi-1}, x_{q}^{(4 \phi-5)}=v$. At phase $\phi$, any process $q$ in $\Pi_{\phi-1}$ either lets $t s_{q}$ unchanged or sets $t s_{q}$ to $\phi$. It follows that $\Pi_{\phi}$ contains $\Pi_{\phi-1}$, and so $\Pi_{\phi}$ is a majority set.

Let $q$ be any process in $\Pi_{\phi}$. We consider two cases.

1. Process $q$ does not execute line 21 at phase $\phi$. Then $t s_{q}^{(\phi)}=t s_{q}^{(\phi-1)}$ and $x_{q}^{(\phi)}=x_{q}^{(\phi-1)}$. It follows that $q$ belongs also to $\Pi_{\phi-1}$. The inductive hypothesis implies that $x_{q}^{(\phi-1)}=v$.

2. Process $q$ updates $t s_{q}$ and $x_{q}$ at phase $\phi$. Let $c^{\prime}=$ $\operatorname{Coord}(q, \phi)$. From lines 17, 18, and 20, it follows that $c^{\prime}$ has casted a vote and vot $e_{c^{\prime}}^{(4 \phi-3)}=x_{q}^{(4 \phi-2)}$. Therefore $c^{\prime}$ has received more than $n / 2$ non-null messages at round $4 \phi-3$. Since each process sends at most one non-null message at this round, one of them has been sent by a process in the majority set $\Pi_{\phi-1}$. Line 12 implies that the largest timestamp received by $c^{\prime}$ at round $4 \phi-3$ is at least equal to $\phi_{1}$. From the inductive hypothesis we derive that vote $e_{c^{\prime}}^{\left(4 \phi_{1}-3\right)}=v$. Hence $x_{q}^{(4 \phi-2)}=v$, as needed.

$\square_{\text {Lemma } 8}$
Let $p^{\prime}$ be a process that decides $v^{\prime}$ at phase $\phi$, and let $c^{\prime}$ denote the coordinator of $p^{\prime}$ at phase $\phi$. By definition of $\phi_{1}$, we have $\phi \geq \phi_{1}$. We are going to prove that $v=v^{\prime}$. For that, we proceed by induction on $\phi-\phi_{1}$.

Basis: $\phi=\phi_{1}$. Process $c^{\prime}$ has necessarily received more than $n / 2$ acknowledgements at phase $\phi=\phi_{1}$. Since each process sends at most one such message per phase, we have $c=c^{\prime}$, and so

$v^{\prime}=\operatorname{vote}_{c^{\prime}}^{\left(4 \phi_{1}-3\right)}=\operatorname{vote}_{c}^{\left(4 \phi_{1}-3\right)}=v$

Inductive step: Let $\phi>\phi_{1}$ and assume that any decision value at phases $\phi_{1}, \ldots, \phi-1$ is equal to $v$. Process $c^{\prime}$ has definitely casted a vote for $v^{\prime}$ at round $4 \phi-1$, and so more than $n / 2$ processes $q$ set $x_{q}$ to $v^{\prime}$ and $t s_{q}$ to $\phi$ at round $4 \phi-2$. Such processes share the same coordinator, namely $c^{\prime}$. It follows that $c^{\prime}$ has received more than $n / 2$ non-null messages at round $4 \phi-3$. By point (1) in Lemma 8 , at least one of them has been sent by some process in $\Pi_{\phi}$. From line 12 and point (2) in Lemma 8, it follows that vote $e_{c^{\prime}}^{(4 \phi-3)}=v$.

Rotating coordinator: Similarly to CoordUniformVoting, we can use the rotating coordinator strategy to determine coordinators in LastVoting: we denote by LastVoting ${ }^{r c}$ the resulting algorithm. With such a coordinator strategy, the existence of a uniformly and well coordinated phase is ensured by the following communication predicate $\mathcal{P}_{\text {Last Voting }}{ }^{\text {rc }}$ :

$\mathcal{P}_{\text {LastVoting }}{ }^{r c}:: \exists \phi_{0}>0: \bigcap_{i=1}^{n} K\left(\phi_{0}+i\right) \neq \emptyset$.

Observe that when (i) choosing the off-line strategy of the rotating coordinator in LastVoting, and (ii) requiring that the condition at line 11 always holds (which means that the coordinator sends a vote in every phase, see line 18), the resulting algorithm, denoted $C T$, corresponds to the Rotating Coordinator algorithm described in [5] for solving Consensus with the failure detector $\diamond \mathcal{S}$.

Because of point (ii), it turns out that $C T$ is safe only under some non-trivial invariant property of the heard-of sets, namely the no split predicate $\mathcal{P}_{\text {nosplit }}$. More precisely, agreement may be violated if two coordinators receive messages from disjoint sets of processes (i.e., there is no process heard by both). This point is not discussed in [5] because the authors assume no link failure and a majority of correct processes, which guarantees $\mathcal{P}_{H O}^{m a j}$, and so $\mathcal{P}_{\text {nosplit }}$. If this assumption does not hold, then the Rotating Coordinator algorithm blocks forever, which is translated in the $\mathrm{HO}$ model by the fact $C T$ is not safe. ${ }^{8}$ The failure detector $\diamond \mathcal{S}$ or

\footnotetext{
8 At two places in each phase, processes in the Rotating Coordinator algorithm wait for at least $n / 2$ messages to advance to the next round. In the HO model, advancing from one round to the next is automatic,
} 
the predicate $\mathcal{P}_{\diamond \text { nek }}$ play a role only for the termination condition of Consensus. Obviously, the notion of failure detectors makes no sense in the context of link failures. However, our remark shows that basically, the Rotating Coordinator algorithm does not tolerate link failures, and more generally dynamic failures. To make it safe in the presence of such failures-which is a quite reasonable requirement-, it is sufficient just to add the test "number of $\langle\nu, \theta\rangle$ received $>n / 2$ " at line 11 .

\subsection{A non-coordinated algorithm without $\mathrm{HO}$ invariant}

The DLS and the LastVoting algorithms show that, if we resort to coordinators, Consensus can be solved without invariant properties for the heard-of sets, i.e., communication predicates of the form:

$\mathcal{P}_{(\square \mathcal{C})}:: \forall r \geq 0: \mathcal{C}$ holds at $r$,

where $\mathcal{C}$ is a predicate over the heard-of sets at a given round (e.g., $\mathcal{P}_{\text {nekrounds }}$ or $\mathcal{P}_{\text {nosplit }}$ ). This naturally leads us to question whether Consensus is solvable without both (i) invariant properties for the HO's and (ii) coordinators. As we shall show below, the answer is yes if there exist rounds in which heard-of sets have a membership larger than $2 n / 3$ (Algorithm 9). We leave the question open in the case heard-of sets are only majority sets.

For that, we design an $\mathrm{HO}$ algorithm that we call OneThirdRule (Algorithm 9). A similar algorithmical schema is used in the first round of [3], in [35], and in the fast rounds of Fast Paxos [28]. Each phase of the OneThirdRule algorithm consists of one single round. Safety conditions of Consensus, namely integrity and agreement, are always satisfied: if some process decides $v$ at line 10 of round $r$, then in any round $r^{\prime} \geq r$, only $v$ can be assigned to any $x_{p}$, and hence only $v$ can be decided. Liveness is ensured by the following condition:

$$
\begin{aligned}
& \exists r_{0}>0, \exists \Pi_{0} \text { s.t. }\left|\Pi_{0}\right|>2 n / 3, \forall p \in \Pi: \\
& \quad\left(H O\left(p, r_{0}\right)=\Pi_{0}\right) \wedge\left(\exists r_{p}>r_{0}:\left|H O\left(p, r_{p}\right)\right|>2 n / 3\right) .
\end{aligned}
$$

The first part, namely the existence of some uniform round $r_{0}$ with an enough large kernel, makes the system "space uniform" in the sense that at the end of round $r_{0}$, all processes adopt the same value for $x_{p}$. The second part of the condition forces every process $p$ to make a decision at the end of round $r_{p}$. These observations establish the following result:

Theorem 8 The HO machine consisting of the OneThirdRule algorithm and the communication predicate $\mathcal{P}_{\left(\mathcal{C}_{0}\right)^{\infty}}$,

and so is not under the control of processes. This is why executions of the Rotating Coordinator algorithm that block (because some process does not eventually hear of a majority of processes) are translated in the HO model into unsafe executions of the $C T$ algorithm. where $\mathcal{C}_{0}$ holds at round $r_{0}$ if

$\exists \Pi_{0}$ s.t. $\left|\Pi_{0}\right|>2 n / 3, \forall p \in \Pi: H O\left(p, r_{0}\right)=\Pi_{0}$,

solves Consensus.

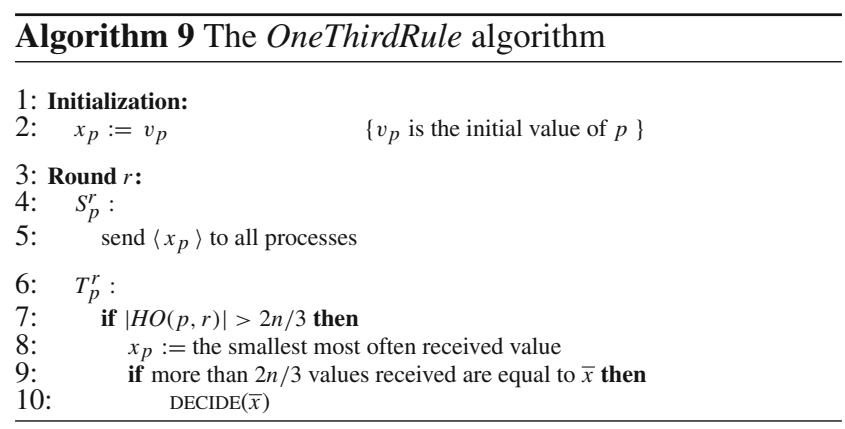

Note that, contrary to all the algorithms we have described up to now, a decision is possible in just one round: if all the initial values are identical ${ }^{9}$ and few transmission faults occur at the beginning (i.e., $\mathcal{C}_{0}$ holds at round 1 ), then every process decides at the end of the first round. Hence, the OneThirdRule algorithm which is a very simple algorithm that does not require any coordinator election procedure, may be quite efficient. Furthermore, the condition for its correctness is satisfied in many real systems. Indeed, one uniform round and "not too many" transmission faults (i.e., heard-of sets with a cardinality greater than $2 n / 3$ ) in some subsequent round is sufficient to entail decision. This may seem to be a strong condition in the classical context of permanent and static failures, such as crash failures, but it is a realistic assumption in systems with transient failures (e.g., crash recovery). For all these reasons, we think that it would be very interesting to develop this solution in many real applications requiring Consensus among processes.

\subsection{Summary}

Table 2 summarizes the various $\mathrm{HO}$ and $\mathrm{CHO}$ Consensus algorithms described in this section, with their correctness requirements. Because of time invariance (cf. Sect. 2.1), the corresponding communication predicates guarantee conditions on rounds that must hold infinitely often, but not necessarily permanently, at least for the last four algorithms. The latter solutions are quite relevant in practice, since one can observe that real systems alternate between "bad" and "good" periods. Note that some of these requirements capture conditions that in the past have been expressed in terms of failure detectors, requiring conditions to eventually hold forever, and thus suitable for permanent failures only.

\footnotetext{
${ }^{9}$ In the context of Atomic Broadcast, messages can sometimes be spontaneously ordered, which translates into identical initial values for Consensus.
} 
Table $2 \mathrm{HO}$ and CHO Consensus algorithms and their correctness conditions with communication predicates

\begin{tabular}{|c|c|c|}
\hline Algorithm & Predicate for safety & Predicate for liveness \\
\hline UniformVoting & no split rounds & $\forall r>0, \exists r_{0} \geq r: r_{0}$ is uniform \\
\hline CoordUniformVoting & no split rounds & $\forall \phi>0, \exists \phi_{0} \geq \phi: \phi_{0}$ is uniformly and well coordinated \\
\hline CoordUniformVoting ${ }^{r c}$ & no split rounds & $\begin{aligned} \forall \phi>0, \exists \phi_{0} \geq \phi: & \bigcap_{i=1}^{n} K\left(\phi_{0}+i\right) \neq \emptyset \\
& \int \phi_{0} \text { is uniform }\end{aligned}$ \\
\hline CoordDLS & none & $\forall \phi>0, \exists \phi_{0} \geq \phi:\left\{\begin{array}{l}K\left(\phi_{0}\right) \text { is a majority set } \\
\phi_{0} \text { is uniformly and well coordinated }\end{array}\right.$ \\
\hline LastVoting & none & $\forall \phi>0, \exists \phi_{0} \geq \phi:\left\{\begin{array}{l}\forall p \in \Pi, \forall r, \text { s.t. } 4 \phi_{0}-3 \leq r \leq 4 \phi_{0}:|H O(p, r)|>n / 2 \\
\phi_{0} \text { is uniformly and well coordinated }\end{array}\right.$ \\
\hline LastVoting ${ }^{r c}$ & none & $\forall \phi>0, \exists \phi_{0} \geq \phi:\left\{\begin{array}{l}\forall p \in \Pi, \forall r \text {, s.t. } 4 \phi_{0}<r<4\left(\phi_{0}+n\right):|H O(p, r)|>n / 2 \\
\bigcap_{i=1}^{n} K\left(\phi_{0}+i\right) \neq \emptyset\end{array}\right.$ \\
\hline OneThirdRule & none & $\forall r>0, \exists r_{0} \geq r: r_{0}$ is uniform and $\left|K\left(r_{0}\right)\right|>2 n / 3$ \\
\hline
\end{tabular}

\section{Conclusion}

The paper proposes a new computational model for faulttolerant distributed systems, which is suitable for describing benign failures in a unified framework. Apart from allowing concise expressions of Consensus algorithms, the model overcomes the limitations of the traditional approaches by getting rid of two basic principles-independence of synchrony degree and failure model; notion of faulty component-on which previous models are all based. In particular, our approach allows us to handle (1) transient failures and (2) failures that hit all the components of the system (links and processes). By contrast, classical models are limited to static faults both in time and space.

As we have observed, a second key point of the HO model is to make sporadic conditions sufficient to solve Consensus, in contrast to the classical models obtained by "augmenting" asynchronous systems with external devices (like failure detectors [5], or other oracles). Indeed, in such augmented asynchronous systems, only stable properties-i.e., properties that once they hold, hold forever-can be usefull for solving problems like Consensus. In the HO formalism, we can give a precise meaning to the statement "the system works correctly for long enough", and we prove that such sporadic conditions are sufficient to make Consensus solvable whereas in augmented asynchronous models, Consensus requires stable properties of the type "eventually and forever the system behaves correctly".

Besides, it is striking to see how, by removing the barrier between synchrony degree and failure model, the HO formalism enables us to give direct proofs of important results in fault-tolerant distributed computing. In this way, we can unify results for synchronous and asynchronous systems, and give a simple proof of the weakest predicate that makes Consensus solvable under some fault bounds.
In this paper, we dealt with benign failures only, but the $\mathrm{HO}$ model can be extended to handle more severe faults. Indeed, we pursue our approach in a sequel paper [2] where we show how to cope with value faults: messages may be corrupted, i.e., at any round $r$, the message received by process $q$ from $p$ may be different of the message that $p$ ought to send to $q$. This novel framework covers the classical Byzantine failures [34] as well as the dynamic transmission faults studied in $[37,38]$. Thus, we derive new Consensus algorithms tolerating both benign and value faults, be they static or dynamic, permanent or transient. Besides, it would be worth extending the notion of translation introduced in Sect. 3 to the HO model with value failures, and studying the connection with the translations of Neiger and Toueg [33] from Byzantine to benign failures in synchronous systems.

Acknowledgments We would like to thank M. Biely, M. Hutle, U. Schmid and J. Widder for many helpful discussions related to the HO model. We also thank L. Lamport, J. Welch, O. Rütti, K. Peters, Ph. Küfner, and U. Nestmann for their comments on an earlier version of the paper, and the anonymous referees.

\section{References}

1. Ben-Or, M. : Another advantage of free choice: Completely asynchronous agreement protocols. In: Proceedings of the Second ACM Symposium on Principles of Distributed Computing, pp. 27-30 (1983)

2. Biely, M., Charron-Bost, B., Gaillard, A., Hutle, M., Schiper, A., Widder, J.: Tolerating corrupted communications. In: Proceedings of the Twentysixth ACM Symposium on Principles of Distributed Computing, pp. 244-253 (2007)

3. Brasileiro, F., Greve, F., Mostéfaoui, A., Raynal, M.: Consensus in one communication step. In: 6th International Conference Parallel Computing Technologies (PaCT), pp. 42-50. Springer, LNCS $2127(2001)$

4. Chandra, T.D., Hadzilacos, V., Toueg, S.: The weakest failure detector for solving consensus. J. ACM 43(4), 685-722 (1996) 
5. Chandra, T.D., Toueg, S.: Unreliable failure detectors for asynchronous systems. J. ACM 43(2), 225-267 (1996)

6. Chandy, K.M., Misra, J.: How processes learn. Distrib. Comput. 1(1), 40-52 (1986)

7. Charron-Bost, B., Hutle, M., Widder, J.: In search of lost time. Technical Report LSR/2008-006, EPFL (2008)

8. Charron-Bost, B., Merz, S.: Formal verification of a Consensus algorithm in the Heard-Of model. TR 20009/07, LIX (2009)

9. Chlebus, B.S., Dicks, K., Pelc, A.: Broadcasting in synchronous networks with dynamic faults. Networks 27, 309-318 (1996)

10. Chor, B., Dwork, C.: Randomization in byzantine agreement. Adv. Comput. Res. 5, 443-497 (1989)

11. Dobrev, S.: Computing input multiplicity in anonymous synchronous networks with dynamic faults. J. Discrete Algorithms 2(4), 425-438 (2004)

12. Dolev, D.: The Byzantine generals strike again. J. Algorithms 3(1), 14-30 (1982)

13. Dolev, D., Dwork, C., Stockmeyer, L.: On the minimal synchronism needed for distributed consensus. J. ACM 34(1), 77-97 (1987)

14. Dolev, D., Reischuk, R., Strong, H.R.: Early stopping in Byzantine agreement. J. ACM 37(4), 720-741 (1990)

15. Dolev, D., Strong, H.R.: Polynomial algorithms for multiple processor agreement. In: Proceedings of the Fourteenth ACM Symposium on Theory of Computing, pp. 401-407. ACM Press, New York (1982)

16. Dolev, D., Strong, H.R.: Authenticated algorithms for Byzantine agreement. SIAM J. Comput. 12(4), 656-666 (1983)

17. Dwork, C., Lynch, N.A., Stockmeyer, L.: Consensus in the presence of partial synchrony. J. ACM 35(2), 288-323 (1988)

18. Elrad, T.E., Francez, N.: Decomposition of distributed programs into communication-closed-layers. Sci. Comput. Progr. 2(3), April 1982

19. Fischer, M.J., Lynch, N.A.: A lower bound for the time to assure interactive consistency. Inf. Process. Lett. 14, 183-186 (1982)

20. Fischer, M.J., Lynch, N.A., Paterson, M.S.: Impossibility of distributed consensus with one faulty process. J. ACM 32(2), 374382 (1985)

21. Fraigniaud, P., Peyrat, C.: Broadcasting in a hypercube when some calls fail. Inf. Process. Lett. 39(3), 115-119 (1991)

22. Gafni, E.: Round-by-round fault detectors: unifying synchrony and asynchrony. In: Proceedings of the Seventeenth ACM Symposium on Principles of Distributed Computing, pp. 143-152 (1998)

23. Gopal, A., Toueg, S.: Reliable broadcast in synchronous and asynchronous environments (preliminary version). In: Bermond, J.-C., Raynal, M. (eds.) Proceedings of the Third International Workshop on Distributed Algorithms. Lecture Notes on Computer Science, vol. 392, pp. 110-123. Springer, Heidelberg (1989)

24. Herlihy, M., Rajsbaum, S., Tuttle, M.: Unifying synchronous and asynchronous message-passing models. In: Proceedings of the Seventeenth ACM Symposium on Principles of Distributed Computing, pp. 123-132 (1998)
25. Keidar, I., Shraer, A.: Timeliness, failure-detectors, and consensus performance. In: ACM Symposium on Principles of Distributed Computing, pp. 169-178 (2006)

26. Kralovic, R., Kralovic, R., Ruzicka, P.: Broadcasting with many faulty links. In: SIROCCO, pp. 211-222 (2003)

27. Lamport, L.: The part-time parliament. ACM Trans. Comput. Syst. 16(2), 133-169 (1998)

28. Lamport, L.: Fast Paxos. Distrib. Comput. 19(2), 79-103 (2006)

29. Lynch, N.A.: Distributed Algorithms. Morgan Kaufmann, Menlo Park (1996)

30. Malkhi, D., Oprea, F., Zhou, L.: $\Omega$ meets Paxos: leader election and stability without eventual timely links. In: International Conference on Distributeed Computing (DISC), pp. 199-213 (2005)

31. Merritt, M.J.: Unpublished Notes (1985)

32. Moses, Y., Rajsbaum, S.: A layered analysis of consensus. SIAM J. Comput. 31(4), 989-1021 (2002)

33. Neiger, G., Toueg, S.: Automatically increasing the fault-tolerance of distributed algorithms. J. Algorithms 11(3), 374-419 (1990)

34. Pease, M., Shostak, R., Lamport, L.: Reaching agreement in the presence of faults. J. ACM 27(2), 228-234 (1980)

35. Pedone, F., Schiper, A., Urban, P., Cavin, D.: Solving agreement problems with weak ordering oracles. In: Proceedings of the 4th European Dependable Computing Conference (EDCC-4), LNCS-2485, pp. 44-61, Toulouse, France. Springer, Heidelberg (2002)

36. Perry, K.J., Toueg, S.: Distributed agreement in the presence of processor and communication faults. IEEE Trans. Softw. Eng. 12(3), 477-482 (1986)

37. Santoro, N., Widmayer, P.: Agreement in synchronous networks with ubiquitous faults. Theor. Comput. Sci. 384, 232-249 (2007)

38. Santoro, N., Widmayer, P.: Time is not a healer. In: Proceedings of the 6th Symposium on Theor. Aspects of Computer Science, pp. 304-313, Paderborn, Germany (1989)

39. Santoro, N., Widmayer, P.: Distributed function evaluation in the presence of transmission faults. In: SIGAL International Symposium on Algorithms, pp. 358-367 (1990)

40. Santoro, N., Widmayer, P.: Majority and unanimity in synchronous networks with ubiquitous dynamic faults. In: Proceedings of the 12th International Colloquium SIROCCO. Lecture Notes on Computer Science, vol. 3499, pp. 262-276, Mont Saint Michel, France. Springer, Heidelberg (2005)

41. Schmid, U.: How to model link failures: A perception-based fault model. In: IEEE International Conference on Dependable Systems and Networks (DSN), pp. 57-66 (2001)

42. Tsuchiya, T., Schiper, A.: Using bounded model checking to verify consensus algorithms. In: International Conference on Distributeed Computing (DISC), pp. 466-480 (2008) 